\title{
A METHOD FOR SENSOR PLACEMENT TAKING INTO ACCOUNT DIAGNOSABILITY CRITERIA
}

\author{
Abed Alrahim YASSINE，StéPhane PlOIX，JeAn-Marie FLAUS \\ Grenoble - Science pour la Conception, l'Optimisation et la Production, G-SCOP lab \\ Grenoble Institute of Technology, BP 46, Saint Martin d'Heres 38402, France \\ e-mail: abed-alrahim.yassinedg-scop.inpg.fr, \\ \{stephane.ploix, jean-marie.flaus\}@inpg.fr
}

\begin{abstract}
This paper presents a new approach to sensor placement based on diagnosability criteria. It is based on the study of structural matrices. Properties of structural matrices regarding detectability, discriminability and diagnosability are established in order to be used by sensor placement methods. The proposed approach manages any number of constraints modelled by linear or nonlinear equations and it does not require the design of analytical redundancy relations. Assuming that a constraint models a component and that the cost of the measurement of each variable is defined, a method determining sensor placements satisfying diagnosability specifications, where all the diagnosable, discriminable and detectable constraint sets are specified, is proposed. An application example dealing with a dynamical linear system is presented.
\end{abstract}

Keywords: fault diagnosis, diagnosability, sensor placement, structural modelling.

\section{Introduction}

In the scientific literature, many approaches to fault diagnosis have been proposed since 1980. The FDI approach, which focuses on fault detection in dynamical systems, was summarized in (Blanke, Kinnaert, Lunze and Staroswiecki, 2006). Related papers in this journal deal with the design of redundancy relations (Shumsky, 2007) as well as with the use of fuzzy logic (Dalton, Klotzek and Frank, 1999; Koscielny, Syfert and Bartys, 1999; Lopez-Toribio, Patton and Uppal, 1999) and neural networks (Korbicz, Patan and Obuchowicz, 1999; Witczak, 2006). The DX approach focuses on diagnosis reasoning. It is summarized in (Hamscher, Console and De Kleer, 1992). Recently, a bridge approach between FDI and DX was proposed (Cordier, Dague, Lévy, Dumas, Montmain, Staroswiecki and Travé-Massuyès, 2000; Nyberg and Krysander, 2003; Ploix, Touaf and Flaus, 2003). Thus, tools for solving diagnosis problems are now well established. However, designing an efficient diagnosis system does not start after the system design but it has to be done during the system design. Indeed, the performance of a diagnostic system highly depends on the number and location of actuators and sensors. Therefore, designing a system that has to be diagnosed requires not only relevant fault diagnosis procedures, but also efficient sensor placement algorithms.

Madron and Veverka (1992) proposed a sensor placement method which deals with a linear system. This method makes use of the Gauss-Jordan elimination to find a minimum set of variables to be measured. This ensures the observability of variables while simultaneously minimizing the cost of sensors. In this approach, the observable variables include the measurable variables plus the unmeasured but deductible variables. Another method of sensor placement was proposed in (Maquin, Luong and Ragot, 1997). This method aims at guaranteeing the detectability and isolability of sensor failures. It is based on the concept of the redundancy degree in variables and on the structural analysis of the system model. The sensor placement problem can be solved by an analysis of a cycle matrix or by using the technique of mixed linear programming. Commault, Dion and Yacoub Agha (2006) proposed an alternative method of sensor placement where a new set of separators (irreducible input separators), which generates sets of system variables in which additional sensors must be implemented to solve the considered problem, is defined.

However, all these methods are not suitable for the design of systems that include a diagnosis system because, 
in this context, the goal of sensor placement should be to make it possible to monitor hazardous components. The sensor placement algorithm should compute solutions that satisfy detectability and diagnosability properties where detectability is the possibility of detecting a fault on a component and diagnosability is the possibility of isolating a fault on a component without ambiguities with any other faulty components. Few methods have focused on this problem.

Travé-Massuyès, Escobet and Milne (2001) proposed a method based on consecutive additions of sensors, which takes into account diagnosability criteria. The principle of this method is to analyze the physical model of a system from a structural point of view. This structural approach is based on Analytical Redundancy Relations (ARRs) (Blanke et al., 2006). However, this method requires an a priori design of all the ARRs for a given set of sensors. Recently, Frisk and Krysander (2007) proposed an efficient method based on a Dulmage-Mendelsohn decomposition (Dulmage and Mendelsohn, 1959; Pothen and Chin-Ju, 1990). Nevertheless, this method only applies to just-determined sets of constraints while most practical systems are under-determined when sensors are not taken into account and over-determined afterwards.

This paper presents a new sensor placement algorithm that takes into account detectability and diagnosability specifications. It applies to systems for which only the structure is known. Thanks to this algorithm, sensor placements satisfying diagnosability objectives can be computed without designing all the ARRs, which is still an open problem. It applies to any system described structurally and does not assume just-determination. Section 2 details the main concepts that are useful to model systems for sensor placement. Then, Section 3 presents how the sensor placement problem is formulated. Section 4 introduces tools for analyzing structural matrices. These tools are then used in Section 5 to determine diagnosability properties directly from the analysis of structural matrices. Section 6 proposes basic algorithms for extracting blocks with useful properties from structural matrices, and Section 7 shows how to use these algorithms to compute sensor placements that satisfy diagnosability specifications. Finally, Section 8 presents an application to an electronic circuit.

\section{System modelling for sensor placement}

Let us introduce the concepts and the formalism used in the paper to formalize the sensor placement problem. Behavioural knowledge starts with phenomena. A phenomenon is a potentially observable element of information about the actual state of a system. It is modelled by an implicitly time-varying variable, which has to be distinguished from a parameter that is model-dependent. Generally speaking, even if a phenomenon is observable, it is not possible to merge it with data because in fault diagnosis data are only known provided that some actuators or sensors behave properly. Phenomena $V(t)=$ $\left\{\ldots, v_{i}(t), \ldots\right\}$ are linked to a phenomenological space $\mathcal{F}(T, V)=\{V(t) ; t \in T\}$, where $T$ stands for a continuous or discrete time set. At any given time $t$ in $T$, these phenomena belong to a domain $\operatorname{dom}(t, V)=\operatorname{dom}(V(t))$ representing all the possible values that the phenomena may have. Consequently, when considering all $t \in T$, $\{\operatorname{dom}(V(t)) ; t \in T\}$ represents a tube in the timed phenomenological space $\mathcal{F}(T, V)$.

All the phenomena have thus to be considered as unknown because observable phenomena are not observations. Let us introduce the concept of a data flow to model actual data recorded on a system. A data flow models data provided by a source of information concerning a phenomenon. A data flow concerning a phenomenon $v$ is denoted by $\operatorname{val}(t, v)$ with $\operatorname{val}(t, v) \in$ $\operatorname{dom}(t, v)$. It corresponds to a trajectory belonging to the tube $\{\operatorname{dom}(t, v) ; t \in T\}$ (see Fig. 1). When information about $v$ is coming from different sources, the different data flows can be denoted by $v l_{i}(t, v)$. Formally, a data flow provided by a component $c$ can be linked to a phenomenon: $o k(c) \rightarrow \forall t \in T, \operatorname{val}(t, v)=v$, which means that if the component named $c$ is in the mode $o k$, then the data $\operatorname{val}(t, v)$ correspond to the actual value of the phenomenon $v$ at any time $t \in T$.

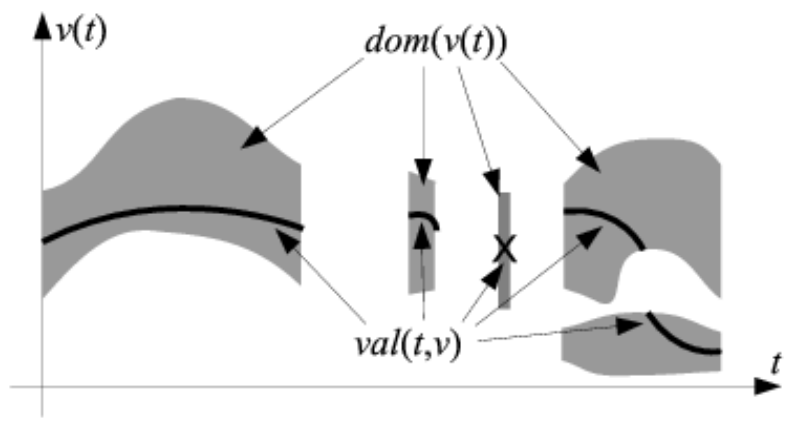

Fig. 1. Tube modelling a variable and a related observation.

In fault diagnosis, a system is not supposed to remain in a given mode. Indeed, diagnostic analysis aims at retrieving the actual behavioral modes of the components of a system. At minimum, two modes are defined: the $o k$ mode, which corresponds to the expected normal behavior, and the $c f$ mode, which is the complementary fault mode: it refers to all the behaviours that do not fit to the expected normal behavior. Sometimes, specific fault modes may be modelled (de Kleer and Williams, 1992; Struss, 1992). They are denoted by a specific label, e.g., the leak mode. Consider, e.g., a pipe where $o k$ and leak are modelled. It yields Modes (pipe) $=$ $\{$ ok, leak, $c f\}$, where $c f$ (pipe) refers to the behaviours 
that do not correspond to ok(pipe) or to leak(pipe).

Except for the complementary fault mode, behavioural modes are modelled by cause-effect relationships between phenomena, which are represented by constraints. Each constraint refers to a set of mappings containing unknown variables and known data flows. Generally speaking, a mapping over $\operatorname{dom}(t, V)$ is defined from one subspace $\operatorname{dom}\left(t, V_{1}\right)$ to another $\operatorname{dom}\left(t, V_{2}\right)$, where $\left\{V_{1}, V_{2}\right\}$ is a partition of $V$. Note that several mappings $\kappa_{i}$ may model the same constraint $k$. If $\kappa_{i}: \operatorname{dom}\left(t, V_{1}\right) \mapsto$ $\operatorname{dom}\left(t, V_{2}\right)$ is a mapping representing a constraint $k$ that models, for example, a component $c_{1}$ in mode $\operatorname{mode}_{1}$ and a component $c_{2}$ in mode mode $_{2}$, we have

$$
\begin{array}{r}
\operatorname{mode}_{1}\left(c_{1}\right) \wedge \operatorname{mode}_{2}\left(c_{2}\right) \rightarrow \\
V_{2}=\kappa_{i}\left(t, V_{1}, \operatorname{val}\left(V_{3}\right)\right) ; \\
V_{1} \in \operatorname{dom}\left(t, V_{1}\right), \quad V_{2} \in \operatorname{dom}\left(t, V_{2}\right),
\end{array}
$$

where the data flow $\operatorname{val}\left(V_{3}\right)$ is considered as being included in the mapping.

But constraint is not strictly equivalent to mapping. A constraint corresponds to a set of equivalent mappings. Firstly, although mappings to multidimensional spaces could be used, they are difficult to manage. It is better to break them down into one-dimensional mappings. In the following, one-dimensional mappings modelling a constraint $k$ are named realizations of $k$. Moreover, several realizations of a constraint may be equivalent. Let $\kappa_{i}$ be a realization from $V \backslash\{v\}$ to $\{v\}$. There may be equivalent realizations defined on $V$ that also model the constraint. Therefore, the notion of constraint can be extended to represent all the equivalent realizations representing a given subset of $\operatorname{dom}(V)$. In the following, a constraint $k$ will be understood as a set of equivalent realizations. It is summarized by the set of variables occurring in the realizations: $\operatorname{var}(k)$. It is assumed that if $k$ is a constraint, for all $v \in \operatorname{var}(k)$, there is an equivalent realization $\kappa_{i}: \operatorname{dom}(t, \operatorname{var}(k) \backslash\{v\}) \mapsto \operatorname{dom}(t, v)$.

To summarize, a system $\Sigma$ is composed of a set of constraints $K_{\Sigma}$ and a set of behavioural modes $\operatorname{Modes}(\Sigma)$ related to components in $\Sigma$. $\operatorname{var}\left(K_{\Sigma}\right)$ is the set of variables, named port in (Chittaro and Ranon, 2004), which models observable phenomena involved in $\Sigma$. Indeed, by extension, the set of variables appearing in a set of constraints $K$ is denoted by $\operatorname{var}(K)=\bigcup_{k \in K} \operatorname{var}(k)$. Each constraint $\kappa \in K_{\Sigma}$ is linked to a mode $m \in \operatorname{Modes}(\Sigma)$ by a first order relationship: $m \rightarrow \kappa$. For the sake of simplicity, in this paper, it is assumed that:

- only ok modes are considered in the sensor placement,

- each constraint $\kappa \in K_{\Sigma}$ models one mode and, conversely, that a mode can be modelled by at most one constraint.
The sensor placement problem then consists in defining the variables of $\operatorname{var}(\Sigma)$ that have to be measured to facilitate the detection and identification of $o k$ modes from Modes $(\Sigma)$. These modes are denoted by $\operatorname{Modes}^{o k}(\Sigma)$. From a mathematical point a view, it is a kind of combinatorial problem. The next section proposes a precise problem formulation.

\section{Problem formulation}

Let us present an intuitive formulation of the problem. Full definitions are given afterwards. The solving of a diagnostic problem is generally decomposed into two consecutive steps. The conflict or symptom generation, also called fault detection in the automatic control community, and the diagnostic analysis, also called fault isolation. The first step relies on consistency tests among minimal testable subsets of constraints ${ }^{1} K \in K_{\Sigma}$ that include data flows (often called OBS for observations). Let $\mathbb{K}$ be the set of minimal testable subsets of constraints. If $K \in \mathbb{K}$ is a set of constraints leading to a test which is inconsistent, this means that, at least, one of the modes corresponding to the constraints of $K$ is not actual. It is therefore important to trace the constraints belonging to a minimal testable subset $K$ because this makes it possible to solve the second sub-problem: the diagnostic analysis, which provides global conclusions in terms of modes about the actual system states. The performance of a diagnostic system is highly dependent on the set $\mathbb{K}$ and, consequently, dependent on the set $K_{\Sigma}$, which highly depends on the dataflows, i.e., on the observations. Additional sensors lead to addtional constraints in $K_{\Sigma}$ and, therefore, to new sets in $\mathbb{K}$. $\mathbb{K}$ can be obtained from combinations of constraints from $K_{\Sigma}$ using possible conflict generation (Pulido and Alonso, 2002), a bipartite graph (Blanke et al., 2006), the Dulmage-Mendelsohn decomposition (Krysander, Aslund and Nyberg, 2008) or elimination rules (Ploix, Désinde and Touaf, 2005). Basically, once $\mathbb{K}$ has been generated, it is possible to compute the performance of the diagnostic system in terms of detectability, discriminability or discernability, and diagnosability. Irrespective of whether or not the performance satisfies the requested performance requirements, the set $K_{\Sigma}$ is modified and the process is conducted once again until the requested performance is reached. However, this process requires lots of computations because the generation of $\mathbb{K}$ is time consuming. Moreover, up to now, no one of these algorithms has been proved to be complete.

Another approach to sensor placement is proposed in this paper. It does not require the computation of $\mathbb{K}$ from $K_{\Sigma}$. It directly solves the following problem by studying the structure of $\Sigma$ : Let $K_{\Sigma}$ be a set of constraints modeling the $o k$ modes of a system $\Sigma$. Let $\operatorname{var}\left(K_{\Sigma}\right)$ be the

\footnotetext{
1 'Minimal' means that to be able to carry out a consistency test, no constraint can be removed from a subset.
} 
variables appearing in $K_{\Sigma}$. The problem to be solved is as follows: What are the complementary constraints modelling sensors dedicated to variables from $\operatorname{var}\left(K_{\Sigma}\right)$ that have to be added to satisfy requested diagnosability performance requirements?

Let us precise the problem formulation by defining the concept of a testable subset or a subsystem (TSS) of constraints and its relationship with the concept of the ARR.

Definition 1. Let $K$ be a set of constraints and $v$ a variable in $\operatorname{var}(K)$ characterized by its domain $\operatorname{dom}(v) . K$ is a solving constraint set for $v$ if, using $K$, it is possible to instantiate $v$ with a value set $S$ such that $S \subset \operatorname{dom}(v)$. A solving constraint set for $v$ is minimal if there is no subset of $K$, which is also a solving constraint set for $v$. A minimal solving constraint set $K$ for $v$ is denoted by $K \vdash v$.

Definition 2. Let $K$ be a set of constraints. $K$ is testable if and only if there is a partition $\left\{K_{1}, K_{2}\right\}$ of $K$ and a variable $v \in \operatorname{var}(K)$ such that $K_{1} \vdash v$ and $K_{2} \vdash v$. If this property is satisfied, it is indeed possible to check if the value set $S_{1}$ deduced from $K_{1}$ is consistent with the value set $S_{2}$ deduced from $K_{2}: S_{1} \cap S_{2} \neq \emptyset$.

Adding any constraint to a testable set also leads to a testable set of constraints. Only minimal testable sets are interesting.

Definition 3. A testable set of constraints is minimal if it is not possible to keep testability when removing a constraint.

A global testable constraint that can be deduced from a TSS is called an analytical relation (ARR). Let $\mathbb{K}_{\Sigma}=\left\{\ldots, K_{k}, \ldots\right\}$ be the set of all the testable subsystems that can be deduced from $K_{\Sigma}$ according to (Blanke et al., 2006; Ploix et al., 2005). Because of the assumed one-to-one relationships between constraints and components, the notions of detectability and discriminability can be extended to constraints.

Definition 4. Let $\mathbb{K}$ be a set of TSSs coming from $\left(K_{\Sigma}, C_{\Sigma}\right)$. A constraint $k \in K_{\Sigma}$ is detectable (Struss, Rehfus, Brignolo, Cascio, Console, Dague, Dubois, Dressler and Millet, 2002) in $\mathbb{K}$ iff $\exists K_{i} \in \mathbb{K} / k \in K_{i}$. By extension, a set of constraints $K \subset K_{\Sigma}$ is detectable in $\mathbb{K}$ if $\forall k_{i} \in K, k_{i}$ is detectable in $\mathbb{K}$.

Definition 5. Two constraints $\left(k_{1}, k_{2}\right) \in K_{\Sigma}^{2}$ are discriminable (Struss et al., 2002) in $\mathbb{K}$ if: $\exists K_{i} \in \mathbb{K} / k_{1} \in$ $K_{i}$ and $k_{2} \notin K_{i}$ or if $\exists K_{j} \in \mathbb{K} / k_{2} \in K_{j}$ and $k_{1} \notin K_{j}$. By extension, the constraints of a set $K \subset K_{\Sigma}$ are discriminable in $\mathbb{K}$ iff $\forall\left(k_{i}, k_{j}\right) \in K^{2}, k_{i}$ and $k_{j}$ are discriminable in $\mathbb{K}$ with $k_{i} \neq k_{j}$.

Obviously, nondetectability implies nondiscriminability.
Definition 6. A constraint $k \in K_{\Sigma}$ is diagnosable (Struss et al., 2002; Console, Picardi and Ribando, 2000) in $\mathbb{K}$ iff it is detectable and $\forall k_{j} \in\left(K_{\Sigma} \backslash k\right),\left(k, k_{j}\right)$ are discriminable in $\mathbb{K}$. By extension, constraints $K \subset K_{\Sigma}$ are diagnosable in $\mathbb{K}$ iff $\forall k_{i} \in K, k_{i}$ are diagnosable in $\mathbb{K}$.

In order to formulate the sensor placement problem, the notion of a terminal constraint has to be introduced.

Definition 7. A terminal constraint $k$ is a constraint that satisfies $\operatorname{card}(\operatorname{var}(k))=1$, where $\operatorname{var}(k)$ is the set of variables appearing in the constraint $k$.

A terminal constraint usually models a sensor or an actuator. It is thus a major concept in sensor placement. Note that if a candidate sensor measures not only one variable $v$ but a combination of several variables $v_{1}, \ldots, v_{n}$, a new constraint $k$ satisfying $\operatorname{var}(k)=\left\{v_{1}, \ldots, v_{n}, v_{*}\right\}$, where $v_{*}$ is a virtual measurable variable, has to be added into $K_{\Sigma}$. Then, the solving is similar to the standard problem.

In fault diagnosis, sensor placement has to satisfy specifications dealing with detectability and diagnosability. Because a one-to-one relation between components and constraints is assumed, what is true for components is also true for constraints. In the following, only constraints will be considered: the analogy with components is implicit. In this paper, complete specifications are considered. Partial specifications can also be managed: they will be presented in a forthcoming paper. These complete specifications consist of a partition of the constraint set $K_{\Sigma}$ into the following subsets:

- the set of constraints $K_{\text {diag }}$ that must be diagnosable,

- the set of subsets of constraints $\mathbb{K}_{\text {nondis }}=$ $\left\{\ldots, K_{i}, \ldots\right\}$ for which each set $K_{i}$ must be nondiscriminable but detectable,

- the set of constraints $K_{\text {nondet }}$ that must be nondetectable,

Complete specifications $K_{\text {diag }}, \mathbb{K}_{\text {nondis }}$ and $K_{\text {nondet }}$ for sensor placement problems are meaningful if the following two properties are satisfied:

1. Sets in specifications must not overlap one another to make sense. Constraint sets have to satisfy $K_{\text {nondet }} \cap$ $K_{\text {diag }}=\phi, \forall K_{i} \in \mathbb{K}_{\text {nondis }}, K_{i} \cap K_{\text {nondet }}=\phi$, $\forall K_{i} \in \mathbb{K}_{\text {nondis }}, K_{i} \cap K_{\text {diag }}=\phi$ and $\forall\left(K_{i}, K_{j}\right) \in$ $\mathbb{K}_{\text {nondis }}^{2}, K_{i} \cap K_{j}=\phi$ if $K_{i} \neq K_{j}$ (no-overlapping property).

2. The union of all the components appearing in $K_{\text {diag }}$, $\mathbb{K}_{\text {nondis }}$ and $K_{\text {nondet }}$ has to correspond to $K_{\Sigma}$ : $K_{\Sigma}=K_{\text {diag }} \cup K_{\text {nondet }} \cup \bigcup_{K_{i} \in \mathbb{K}_{\text {nondis }}} K_{i}$ (completeness property). 
If these properties are satisfied, the complete specifications are qualified as consistent in $K_{\Sigma}$.

Satisfying the diagnosability specifications requires information delivered by sensors. Let $K_{\Sigma}^{\prime}$ represent the system $\Sigma$ with additional sensors where $K_{\Sigma^{\prime}}$ contains the constraints $K_{\Sigma}$ of the system $\Sigma$ plus the additional terminal constraints modelling the additional sensors. Therefore, solving a sensor placement problem consists in determining additional terminal constraints in $K_{\Sigma^{\prime}}$ that lead to the satisfaction of complete specifications.

In the next sections, diagnosability properties of structural matrices are established and used for the design of a sensor placement satisfying diagnosability specifications.

\section{Basic properties of structural matrices}

Before pointing out diagnosability properties, some basic properties of structural matrices have to be established. The constraints $K_{\Sigma}=\left\{\ldots, k_{i}, \ldots\right\}$ can be represented by a structural matrix $\mathcal{M}_{\Sigma}$, which is an incidence matrix representing the mapping $\mathcal{M}_{\Sigma}: \operatorname{var}\left(K_{\Sigma}\right) \rightarrow K_{\Sigma}$.

According to the definition, a TSS is a minimum set of constraints $K$ such that there is at least one variable for which two different minimal solving sets can be found. A minimal solving set leading to a variable $v$ corresponds to a value propagation (Apt, 2003) starting usually, but not necessarily, by terminal constraints and leading to $v$. Therefore, a TSS can also be seen as two distinct value propagations leading to a given variable. This point of view has been adopted as a theoretical tool to develop proofs.

Let $k_{1}$ and $k_{2}$ be two constraints. The propagation of a variable $v$ between $k_{1}$ and $k_{2}$ is possible only if $v \in \operatorname{var}\left(k_{1}\right) \cap \operatorname{var}\left(k_{2}\right)$. The variable $v$ is qualified as propagable between $k_{1}$ and $k_{2}: v$ is a link between $k_{1}$ and $k_{2}$. In the corresponding structural matrix, this link is represented by a thick line:

\begin{tabular}{|l|l|l|}
\hline & $v$ & $\ldots$ \\
\hline$k_{1}$ & $\mathbf{1}^{1}$ & $\ldots$ \\
\hline$k_{2}$ & $\mathbf{1}$ & $\ldots$ \\
\hline
\end{tabular}

Consider now a system defined by $K_{\Sigma}=$ $\left\{k_{1}, k_{2}, k_{3}, k_{4}, k_{5}\right\}$ with $\operatorname{var}\left(k_{1}\right)=\left\{v_{1}, v_{3}\right\}, \operatorname{var}\left(k_{2}\right)=$ $\left\{v_{1}, v_{2}\right\}, \operatorname{var}\left(k_{3}\right)=\left\{v_{2}, v_{3}\right\}, \operatorname{var}\left(k_{4}\right)=\left\{v_{2}\right\}$ and $\operatorname{var}\left(k_{5}\right)=\left\{v_{3}\right\}$. Terminal constraints $k_{4}$ and $k_{5}$ model sensors or actuators. Each terminal constraint contains known data. Figure 2 represents examples of propagations that lead to a TSS with a bipartite graph. But in a bipartite graph, links do not appear clearly: they correspond to alternate paths (or chains) with the pattern 'constraintvariable-constraint'. Links appear more clearly in structural matrices as lines linking two constraints. In the fol-

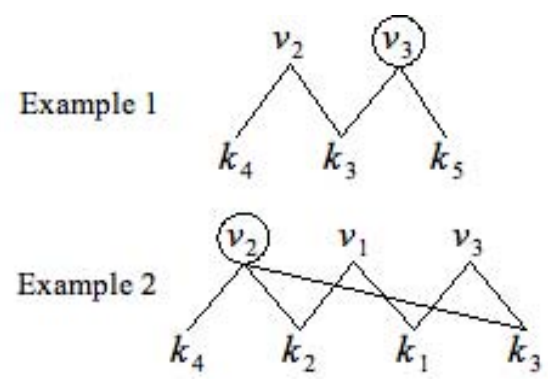

Fig. 2. Link between propagations and minimal testable subsets.

lowing structural matrices, the variables surrounded by a circle represent the variables that can be instantiated twice. The relevance of links remains obvious in Example 2 , where a propagation does not start by a terminal constraint. The paths corresponding to propagations of solving sets were drawn. Variable $v_{2}$ was instantiated twice.

Example 1
\begin{tabular}{|l|l|l|l|}
\hline & $v_{1}$ & $v_{2}$ & $v_{3}$ \\
\hline$k_{1}$ & 1 & & 1 \\
\hline$k_{2}$ & 1 & 1 & \\
\hline$k_{3}$ & & 1 & 1 \\
\hline$k_{4}$ & & 1 & \\
\hline$k_{5}$ & & & 1 \\
\hline
\end{tabular}

Example 2
\begin{tabular}{|l|c|c|c|}
\hline & $v_{1}$ & $v_{2}$ & $v_{3}$ \\
\hline$k_{1}$ & 1 & & 1 \\
\hline$k_{2}$ & 1 & 1 & \\
\hline$k_{3}$ & & 1 & 1 \\
\hline$k_{4}$ & & 1 & \\
\hline$k_{5}$ & & & 1 \\
\hline
\end{tabular}

Once again, paths may be reduced to links (thick lines). The following example points out another structural matrix with two propagations leading to variable $v_{3}$ :

\begin{tabular}{|l|l|l|l|}
\hline & $v_{1}$ & $v_{2}$ & $v_{3}$ \\
\hline$k_{1}$ & 1 & 1 & 1 \\
\hline$k_{2}$ & 1 & $\mathbf{1}$ & \\
\hline$k_{3}$ & & $\mathbf{1}$ & \\
\hline$k_{4}$ & & & 1 \\
\hline
\end{tabular}

The concept of linked constraints has to be formalized because discriminability depends on this concept. Before defining linked constraints, the concept of interconnected constraints has to be introduced. The constraints of a system $\Sigma$ may be modelled by a non-directed bipartite graph $\left(K_{\Sigma}, \operatorname{var}\left(K_{\Sigma}\right), E_{\Sigma}\right)$, where $E_{\Sigma}$ is the set of edges. Each edge $e=(k, v)$ reflects $v \in \operatorname{var}(k)$.

Definition 8. A set of constraints $K \subset K_{\Sigma}$ is interconnected by a set of variables $V \subset \operatorname{var}\left(K_{\Sigma}\right)$ iff there is a tree $(K, V, E) \subset\left(K_{\Sigma}, \operatorname{var}\left(K_{\Sigma}\right), E_{\Sigma}\right)$ with constraints at extremities (see, e.g., (Bollobás, 1998)), which satisfies $\operatorname{card}(V)=\operatorname{card}(K)-1$.

To point out the link with bipartite graph theory, if $K$ is interconnected by $V$ in $K_{\Sigma}, V$ is necessarily a complete 
coupling for $K$ with respect to variables. The notion of a linked set of constraints can now be introduced.

Definition 9. A set of constraints $K \subset K_{\Sigma}$ is linked in $K_{\Sigma}$ by a set of variables $V \subset \operatorname{var}\left(K_{\Sigma}\right)$ iff $K$ is interconnected by $V$ and iff the other constraints of $K_{\Sigma}$ (i.e., $K_{\Sigma} \backslash K$ ) do not contain any variable of $V$. The variables of $V$ are called linking variables for $K$. They are denoted by $\operatorname{var}_{\text {linking }}\left(K, K_{\Sigma}\right)$.

The shape of a structural matrix dealing with linked constraints is drawn in Fig. 3.

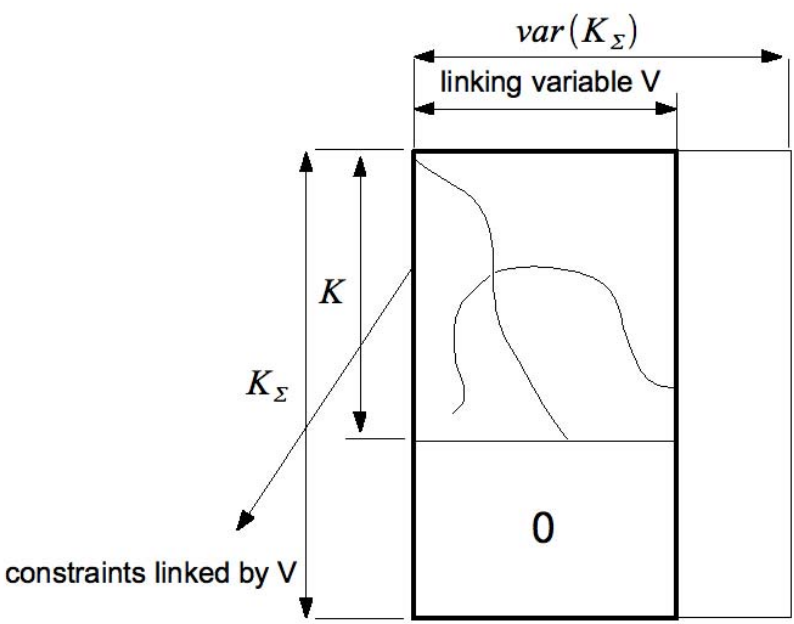

Fig. 3. Structural matrix of a constraint set $K$, which is linked by a set of variables $V$.

The concept of linked constraints is strongly connected with discriminability.

Lemma 1. A set of constraints $K \subset K_{\Sigma}$ linked by a set of variables $V \subset V_{\Sigma}$ is necessarily non-discriminable.

Proof. Indeed, because variables in $V$ only appear in the constraints belonging to $K$, the only way for propagating variables is to use the constraints in $K$ and the variables in $V$. What is more, because there is a tree $(K, V, E) \subset\left(K_{\Sigma}, \operatorname{var}\left(K_{\Sigma}\right), E_{\Sigma}\right)$ with constraints at extremities, instantiating all the variables in $V$ involves at least the achievement of the propagations defined by the tree.

Therefore, all the constraints are invariably found together in the TSS. In order to improve the clarity of these explanations, let us introduce the notion of stump variables.

Definition 10. A set of variables $\operatorname{var}(K)$ appearing in a set of constraints $K$ but not in the other constraints of $K_{\Sigma}$ (i.e., $\left.K_{\Sigma} \backslash K\right)$ are named stump variables in $K_{\Sigma}$ with respect to $K$. They are denoted by $v r_{\text {stump }}\left(K, K_{\Sigma}\right)$.
For instance, the set of variables $V$ that link a set of constraints $K$ belong to the stump variables $\operatorname{var}_{\text {stump }}\left(K, K_{\Sigma}\right)$ with $K \subset K_{\Sigma}$.

A set of constraints cannot be used to generate a TSS if they are linked and if there are additional variables that cannot be propagated. These constraints are qualified as isolated. Detectability depends on this concept.

Definition 11. A set of several constraints $K \subset K_{\Sigma}$ is isolated in $K_{\Sigma}$ by a set of variables $V \subset \operatorname{var}\left(K_{\Sigma}\right)$ if it is linked by $V$ and if there is at least one variable in $\operatorname{var}(K) \backslash V$ that does not belong to other constraints of $K_{\Sigma}$ (i.e., $K_{\Sigma} \backslash K$ ). If the set contains only one constraint, the link condition disappears.

The shape of a structural matrix dealing with isolated constraints is shown in Fig. 4.

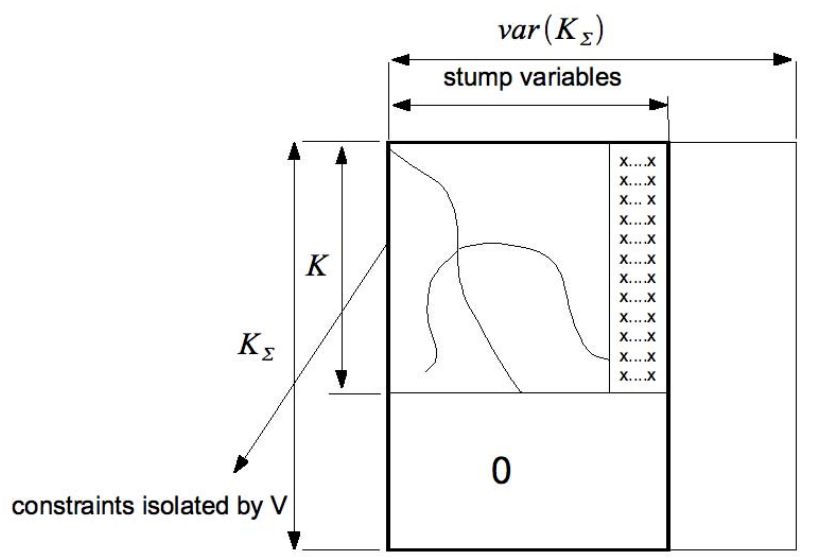

Fig. 4. Structural matrix of a constraint set, which is isolated by the set of variables $V$.

The concept of isolated constraints is strongly linked with detectability.

Lemma 2. A set of constraints $K \subset K_{\Sigma}$ isolated in $K_{\Sigma}$ by $V$ is necessarily non-detectable.

Proof. The constraints $K$ isolated in $K_{\Sigma}$ by $V$ will always come together in the TSS because, by definition, they are linked by $V$. Because of the fact that, in isolated constraints, there is at least one additional variable in $\operatorname{var}(K)$ which does not appear in other constraints (i.e., $K_{\Sigma} \backslash K$ ), it is not possible to instantiate this variable and, therefore, this set of constraints cannot be involved into a TSS: constraints $K$ are thus non-detectable.

\section{Diagnosability properties of structural matrices}

This section aims at setting up a direct link from sets of constraints to detectability and diagnosability properties. 
Firstly, it is obvious that adding additional constraints connected to all the variables $\operatorname{var}(k)$ appearing in a constraint $k$ ensures the diagnosability of $k$.

Lemma 3. Let $k \in K_{\Sigma}$ be a constraint. If additional terminal constraints dealing with all the variables in $\operatorname{var}(k)$ are added, then the constraint $k$ is diagnosable.

Proof. Because there are additional terminal constraints connected to each variable in $V(k)$, a value can be assigned to each variable. Consequently, there is one TSS containing $k$ plus additional terminal constraints connected to variables in $\operatorname{var}(k)$. Therefore, the constraint $k \in K_{\Sigma}$ is necessarily diagnosable because there is one TSS that does not contain other constraints of $K_{\Sigma}$ (i.e., $\left.K_{\Sigma} \backslash\{k\}\right)$.

Lemma 3 can be directly applied to all the constraints of a constraint set.

Corollary 1. If additional terminal constraints dealing with all the variables var $(K)$ of a constraint set $K$ belong to $K_{\Sigma}$, then each constraint $k \in K$ is diagnosable.

In Lemma 2, a relationship between isolated constraints and the detectability property has been presented. The next lemma generalizes the previous results.

Lemma 4. A sufficient condition for a subset of constraints $K \subset K_{\Sigma}$ to be non-detectable is that there is a sequence $\left(K_{1}, \ldots, K_{m}\right)$ of $m$ sets of constraints making up a partition $\mathcal{P}(K)$ of $K$ such that each $K_{i}$ is isolated in $K_{\Sigma} \backslash \bigcup_{j<i} K_{j}$ ( $K_{1}$ is a limit case: it should be isolated in $\left.K_{\Sigma}\right)$.

Proof. The case of $K_{1}$ has been discussed in Lemma 2: because the constraints in $K_{1}$ are isolated in $K_{\Sigma}$, they are non-detectable and therefore cannot be included in the TSS. Then, the remaining candidate constraints for the TSS belong to $K_{\Sigma} \backslash K_{1}$. Because $K_{2}$ is isolated in $K_{\Sigma} \backslash K_{1}$, they are non-detectable. The reasoning can be extended to any $K_{i}$. Consequently, the constraints in $K=\bigcup_{i} K_{i}$ are non-detectable.

Figure 5 indicates the shape of a structural matrix of non-detectable constraints.

Consider, e.g., a system modelled by the following structural matrix:

\begin{tabular}{|c|c|c|c|c|c|c|}
\hline & $v_{1}$ & $v_{2}$ & $v_{3}$ & $v_{4}$ & $v_{5}$ & $v_{6}$ \\
\hline \hline$k_{1}$ & 1 & 0 & 0 & 1 & 0 & 0 \\
\hline$k_{2}$ & 0 & 1 & 1 & 0 & 1 & 0 \\
\hline$k_{3}$ & 0 & 1 & 1 & 0 & 1 & 0 \\
\hline$k_{4}$ & 0 & 0 & 0 & 1 & 0 & 1 \\
\hline$k_{5}$ & 0 & 0 & 0 & 1 & 1 & 1 \\
\hline
\end{tabular}

Assume that the set $K=\left\{k_{1}, k_{2}, k_{3}\right\}$ is required to be non-detectable. In this example, there exists a pair $\left(\left\{k_{1}\right\},\left\{k_{2}, k_{3}\right\}\right)$ such that each element $K_{i}$ satisfies

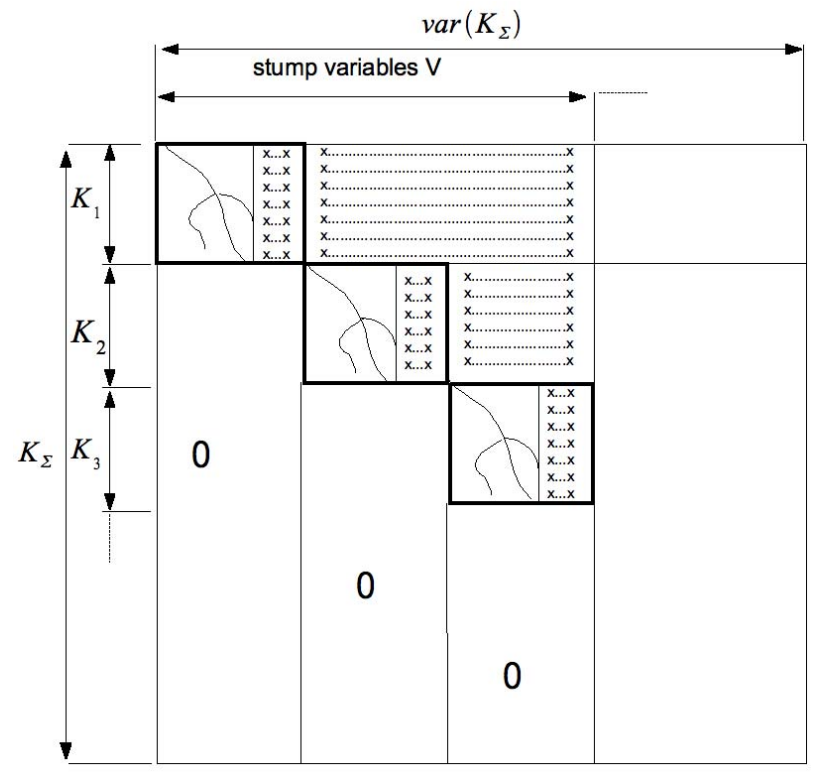

Fig. 5. Structural matrix of non-detectable constraints.

Lemma 4. If there are no additional terminal constraints containing $v_{1}, v_{2}$ and $v_{3}$, the subset $K$ is necessarily nondetectable.

Lemma 5. A sufficient condition for each set $K_{i} \subset K$ belonging to a set of $m$ constraint sets $\mathbb{K}=\left\{K_{1}, \ldots, K_{m}\right\}$ such that $\forall K_{i} \neq K_{j}, K_{i} \cap K_{j}=\emptyset$, to be nondiscriminable is that each $K_{i}$ is linked by a set of variables $V_{i}$.

Proof. This lemma is a direct application of Lemma 1 to several sets of constraints.

Consider, for example, a system modelled by the following structural matrix:

\begin{tabular}{|c|c|c|c|c|c|}
\hline & $v_{1}$ & $v_{2}$ & $v_{3}$ & $v_{4}$ & $v_{5}$ \\
\hline \hline$k_{1}$ & 1 & 0 & 1 & 1 & 1 \\
\hline$k_{2}$ & 1 & 1 & 1 & 1 & 0 \\
\hline$k_{3}$ & 1 & 1 & 1 & 0 & 1 \\
\hline$k_{4}$ & 0 & 1 & 1 & 0 & 0 \\
\hline$k_{5}$ & 0 & 0 & 0 & 1 & 1 \\
\hline
\end{tabular}

Assume that $K=\left\{k_{1}, k_{2}, k_{3}, k_{4}\right\}$ is a constraint subset that should be non-discriminable. Because the constraints $k_{1}, k_{2}, k_{3}$ and $k_{4}$ are linked by $V=\left\{v_{1}, v_{2}, v_{3}\right\}$, Lemma 5 is satisfied. Therefore, $k_{1}, k_{2}, k_{3}$ and $k_{4}$ are nondiscriminable provided that no additional terminal constraints contain a variable of $V$.

The following theorem collects the results of Lemmas 3, 4 and 5 .

Theorem 1. Let $K_{\Sigma}$ be a set of constraints and $K_{\text {nondet, }}$, $\mathbb{K}_{\text {nondis }}$ and $K_{\text {diag }}$ be the specifications of a sensor place- 
ment problem consistent in $K_{\Sigma}$. Sufficient conditions for the specifications to be fulfilled are as follows:

1. There exists a system $\left(K_{1}, \ldots, K_{p}\right)$ of $p$ sets of constraints making up a partition $\mathcal{P}\left(K_{\text {nondet }}\right)$ of $K_{\text {nondet }}$ such that each $K_{i}$ is isolated in $K_{\Sigma} \backslash \bigcup_{j<i} K_{j}$ ( $K_{1}$ is a limit case: it should be isolated in $K_{\Sigma}$ ) as shown in Fig. 5.

2. Each set $K_{i}$ belonging to $\mathbb{K}_{\text {nondis }}=\left\{K_{1}, \ldots, K_{m}\right\}$ such that $\forall K_{i} \neq K_{j}, K_{i} \cap K_{j}=\emptyset$, is linked by $a$ set of variables $V_{i}$ in considering only the detectable constraints $K_{\Sigma} \backslash K_{\text {nondet }}$.

3. Additional terminal constraints are added on the variables

$$
\begin{aligned}
& V_{\text {candidate }} \\
& \quad=\operatorname{var}\left(K_{\Sigma}\right) \backslash\left(\operatorname{var}_{\text {stump }}\left(K_{\text {nondet }}, K_{\Sigma}\right)\right. \\
& \left.\cup \bigcup_{K_{j} \in \mathbb{K}_{\text {nondis }}} \operatorname{var}_{\text {linking }}\left(K_{j}, K_{\Sigma} \backslash K_{\text {nondet }}\right)\right) .
\end{aligned}
$$

Proof. The proof relies on the resulting structure of the structural matrix, which directly stems from Corollary 5 as well as Lemmas 4 and 5. Note that Point 2 could also be stated for the whole set of constraints $K_{\Sigma}$. However, it is not useful to include non-detectable constraints, which will not appear in the resulting TSS: it would be less conservative.

Because of Lemmas 4 and 5, the variables of $\operatorname{var}\left(K_{\text {diag }}\right)$ cannot contain variables appearing in the variables involved in (1) and (2), that is to say, in $\operatorname{var}_{\text {stump }}\left(K_{\text {nondet }}, K_{\Sigma}\right)$ and in $\bigcup_{K_{j} \in \mathbb{K}_{\text {nondis }}} \operatorname{var}_{\text {linking }}\left(K_{j}, K_{\Sigma} \backslash K_{\text {nondet }}\right)$. It follows that $\operatorname{var}\left(K_{\text {diag }}\right)$ satisfies $\operatorname{var}\left(K_{\text {diag }}\right) \subset V_{\text {candidate }}$ Because the variables of $V_{\text {candidate }}$ can be instantiated with measured values, all the constraints of $K_{\text {diag }}$ are diagnosable following Corollary 5.

The point which has to be proved is that, in specifications, $\mathbb{K}_{\text {nondis }}$ defines non-discriminable but detectable sets and not only non-discriminable sets as in Lemma 5: the detectability of sets in $\mathbb{K}_{\text {nondis }}$ has to be proved. The variables $\operatorname{var}\left(K_{i}\right)$ of a constraint set $K_{i} \in \mathbb{K}_{\text {nondis }}$ can be decomposed into two sets: $V_{i}^{-}$and $V_{i}^{+}$, where $V_{i}^{-}=\operatorname{var}_{\text {linking }}\left(K_{i}, K_{\Sigma} \backslash K_{\text {nondet }}\right)$ contains the linking variables and $V_{i}^{+}$contains the remaining variables $V_{i}^{+}=\operatorname{var}\left(K_{i}\right) \backslash V_{i}^{-}$. Lemmas 4 and 5 imply that the set $V_{i}^{+}$cannot contain variables in $\operatorname{var}_{\text {stump }}\left(K_{\text {nondet }}, K_{\Sigma}\right)$ and in $\bigcup_{K_{j} \in \mathbb{K}_{\text {nondis }} ; K_{j} \neq K_{i}} \operatorname{var}_{\text {linking }}\left(K_{j}, K_{\Sigma}\right)$. Therefore, $V_{i}^{+}$satisfies $V_{i}^{+} \subset V_{\text {candidate }}$.

Because of the third point of the theorem, all the variables of $V_{\text {candidate }}$ are known: additional terminal constraints are indeed added, and there is necessarily a TSS dealing with all the constraints in $K_{i}$. It proves that the
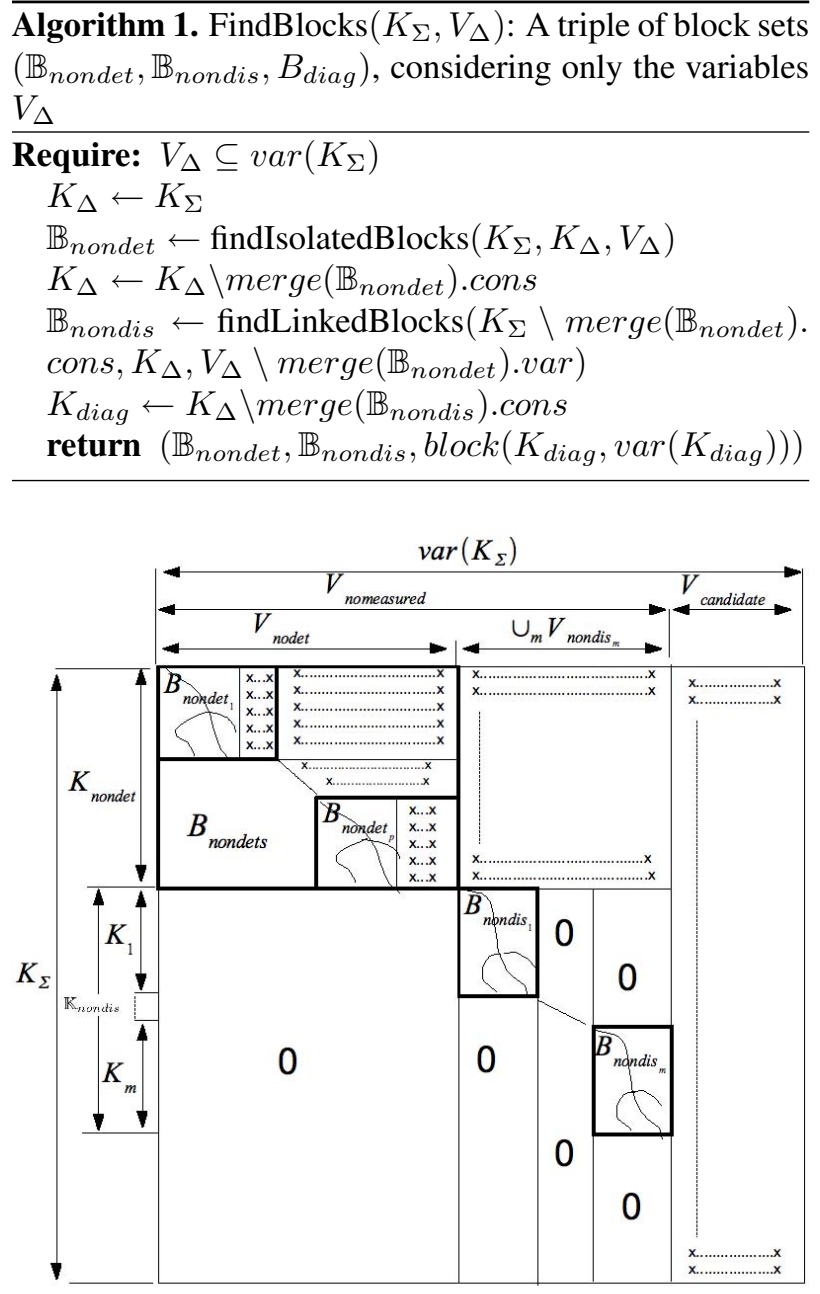

Fig. 6. Shape of a structural matrix satisfying Theorem 1.

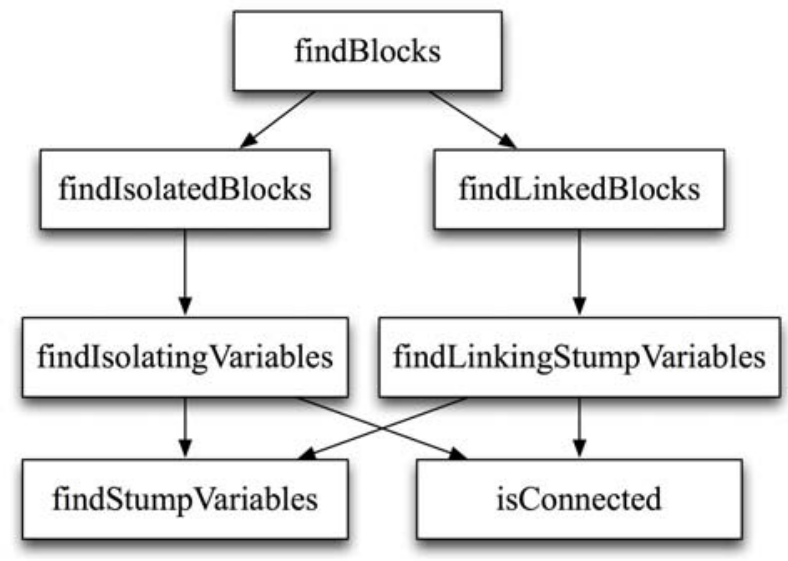

Fig. 7. Dependency scheme of the block extraction algorithm.

constraint set $K_{i}$ is necessarily detectable. Because this result holds for any $K_{i} \in \mathbb{K}_{\text {nondis }}$, it proves the theorem. 


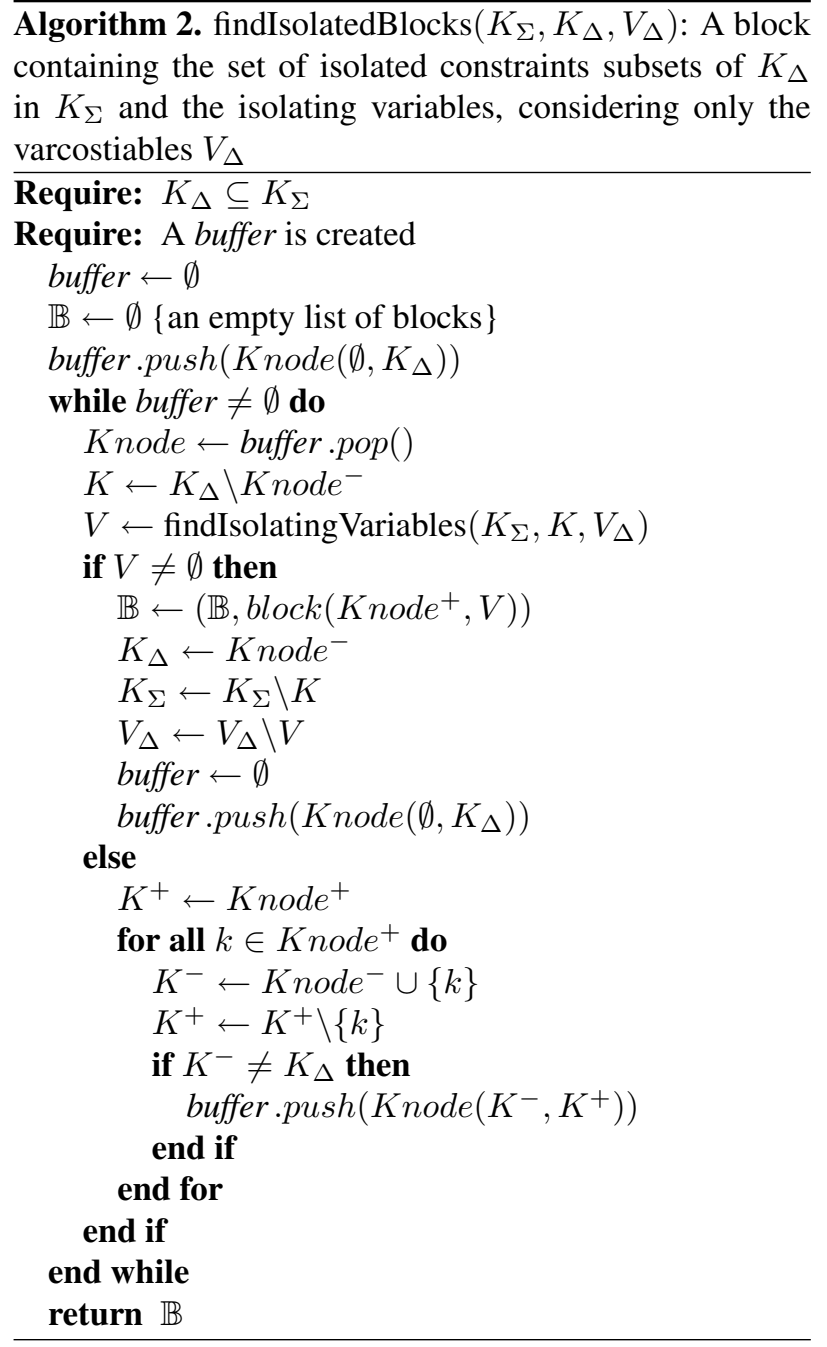

Satisfying the assumptions of Theorem 1 guarantees that the specifications are satisfied. However, because the theorem provides only a sufficient condition for diagnosability, the number of additional terminal constraints is not necessarily minimal. It has to be checked afterwards.

In the next section, an algorithm for extracting blocks from a structural matrix is presented. This algorithm is required by methods for sensor placement based on complete specifications.

\section{Extracting blocks from a structural matrix}

Before presenting an algorithm for extracting blocks from a structural matrix $K_{\Sigma}$, let us introduce some notation. Firstly, the notion of a block is formalized: a block is a couple defined by block $=(K, V)$ where block.cons $=$ $K$ and block.var $=V$ stand respectively for a set of con-

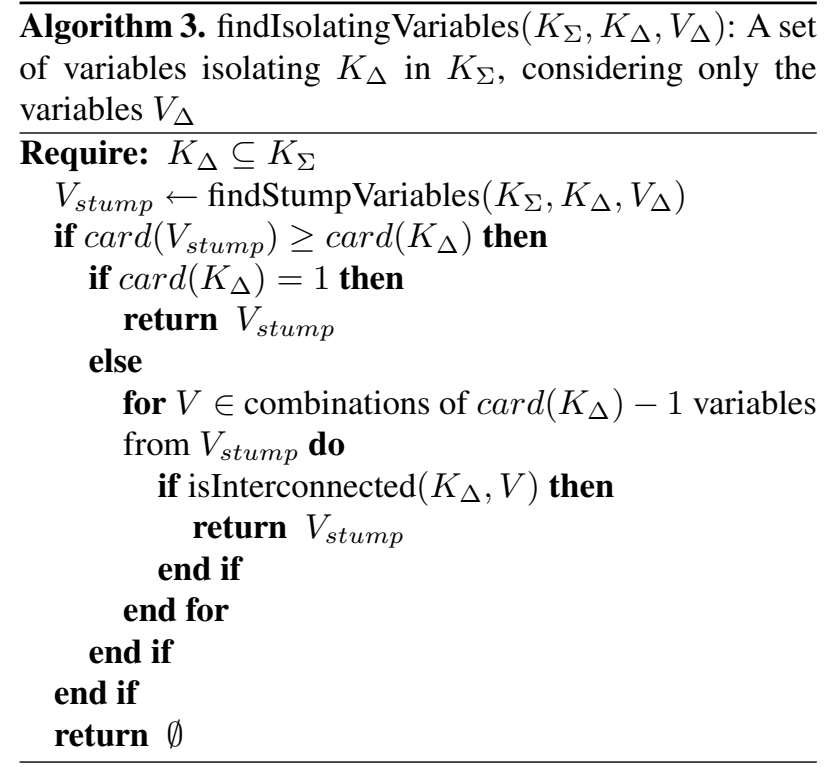

straints and a set of variables. Two blocks can be merged:

$$
\begin{aligned}
& \text { merge }\left(\text { block }_{1}, \text { block }_{2}\right) \\
& =\text { block }\left(\text { block }_{1} \text {.cons } \cup \text { block } k_{2}\right. \text {.cons, }
\end{aligned}
$$

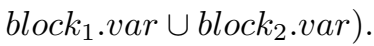

A set of blocks is denoted by the symbol $\mathbb{B}$. By extension, the block resulting from the merging of sets of blocks $\mathbb{B}$ is denoted by $\operatorname{merge}(\mathbb{B})$.

Figure 7 represents the dependency scheme between the methods that are defined. The main algorithm is named findBlocks (Algorithm 1). It extracts the different blocks that appear in Theorem 1, considering only the variables $V_{\Delta}$.

In order to describe the methods findIsolatedBlocks() and findLinkedBlocks(), the notions of Knode and buffer of Knodes are introduced. A Knode is a couple of constraint sets: Knode $=\operatorname{Knode}\left(K^{-}, K^{+}\right)$, where Knode $^{-}=K^{-}$and Knode $^{+}=K^{+}$. A buffer is a special First-In First-Out buffer. The basic functionalities are buffer .push (Knode) and buffer.pop(). They respectively correspond to adding a Knode in the buffer and getting a Knode from the buffer.

Using these notions, the algorithm findIsolatedBlocks() (Algorithm 2) extracts the set of isolated blocks from a set of constraints $K_{\Delta} \subseteq K_{\Sigma}$, considering only the variables $V_{\Delta}$. According to Lemma 4 , the constraints belonging to the resulting blocks are not detectable.

This algorithm depends on the findIsolatingVariables() method. It is given by Algorithm 3.

The algorithm findLinkedBlocks() (Algorithm 4) extracts the set of linked constraints from a set $K_{\Delta} \subseteq K_{\Sigma}$, considering only the variables $V_{\Delta}$. The structure of this algorithm is very closed to that of Algorithm 2. Accord- 
Algorithm 4. findLinkedBlocks $\left(K_{\Sigma}, K_{\Delta}, V_{\Delta}\right)$ : A set of blocks, where each one corresponds to a linked but not isolated set of constraints, and its corresponding linking variables, considering only the variables $V_{\Delta}$

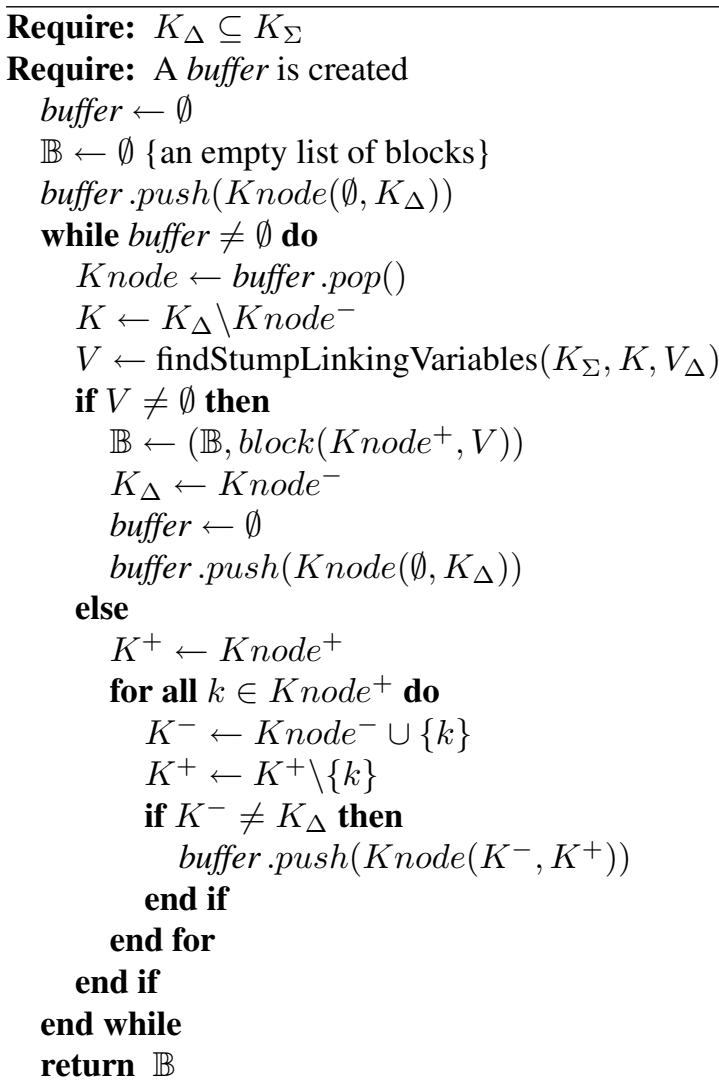

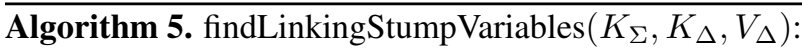
One set of stump variables linking $K_{\Delta}$ in $K_{\Sigma}$, considering only the variables $V_{\Delta}$

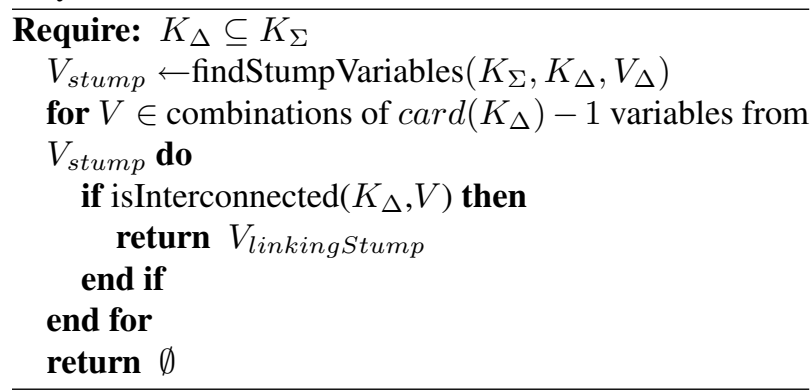

ing to Lemma 5, the constraints belonging to the resulting blocks are not discriminable. This algorithm depends on the findLinkingStumpVariables() method, which is given by Algorithm 5.

Finally, according the Fig. 7, the algorithms findIsolatingVariables() and findLinkedBlocks() depend on two methods findStumpVariables() (Algorithm 6) and isInter-

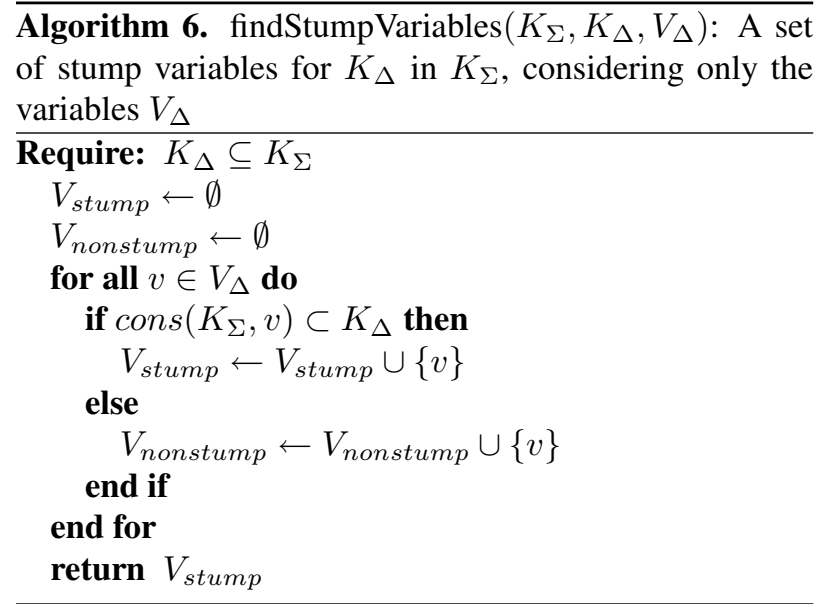

connected() (Algorithm 7).

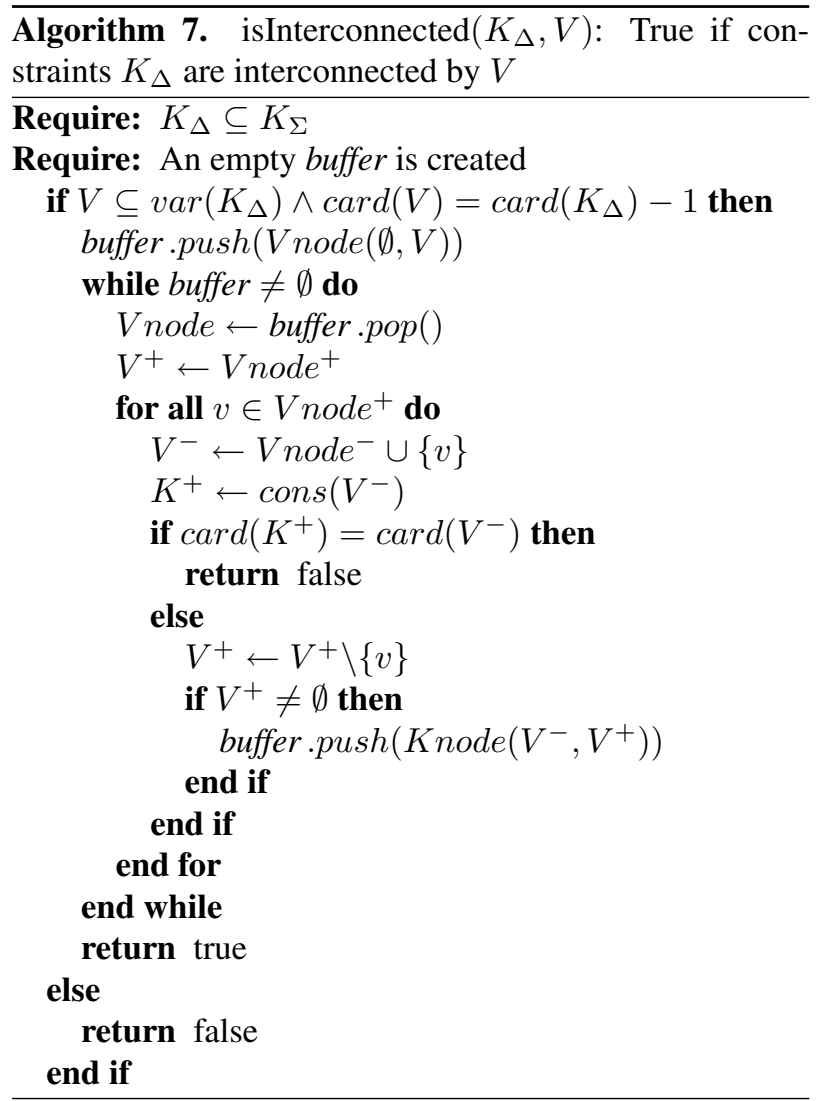

The top-level method findBlocks $\left(K_{\Sigma}\right)$ leads to the blocks depicted in Fig. 6. These results are very useful to support the sensor placement. Indeed, constraints belonging to $B_{\text {diag }}$.cons are already diagnosable. Therefore, finding a sensor placement satisfying the specifications requires that the specified $K_{\text {diag }}^{\text {spec }}$ should include $B_{\text {diag.cons: }}$

$$
B_{\text {diag. }} . \text { cons } \subset K_{\text {diag }}^{\text {spec }}
$$




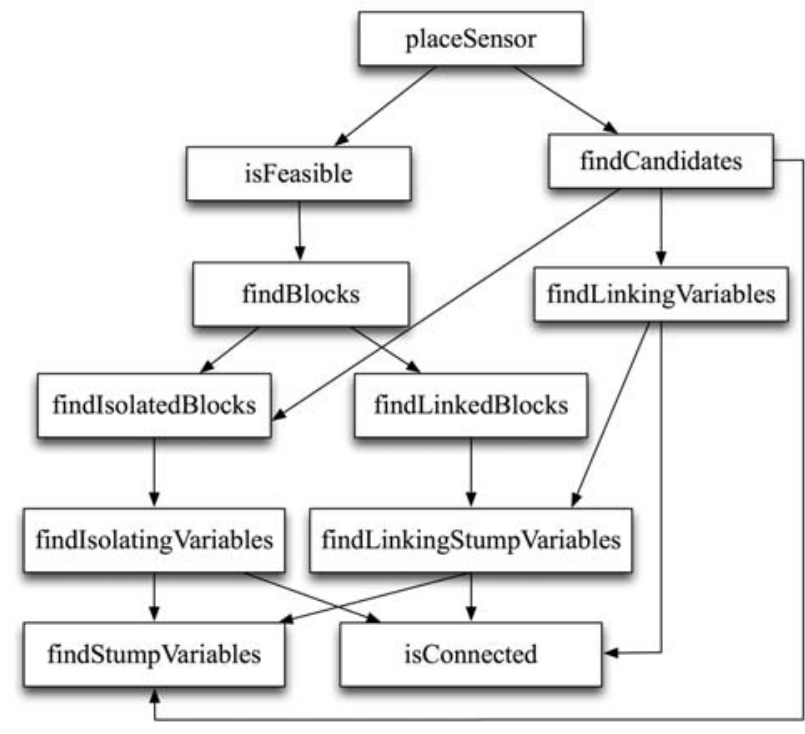

Fig. 8. Dependency scheme of the sensor placement method for complete specifications.

In much the same way, the constraints merge $\left(\mathbb{B}_{\text {nondis }}\right)$.cons $\cup B_{\text {diag. }}$.cons are already detectable. Therefore, finding a sensor placement satisfying the specifications requires that the specified $K_{\text {nondet }}^{\text {spec }}$ should be included in merge $\left(\mathbb{B}_{\text {nondet }}\right)$.cons:

$$
K_{\text {nondet }}^{\text {spec }} \subset \operatorname{merge}\left(\mathbb{B}_{\text {nondet }}\right) \text {.cons. }
$$

\section{Method for sensor placement}

A method for optimal sensor placements satisfying diagnosability specifications is proposed in this section. This method deals with complete specifications: $K_{\text {diag }}^{\text {spec }}$, $\mathbb{K}_{\text {nondis }}^{\text {spec }}$ and $K_{\text {nondet }}^{\text {spec }}$ (see Section 4).

There may be several sensor placements that satisfy diagnosability specifications. In order to select the most interesting one, a criterion based on the cost of the sensor placement is considered. Introduce the following notation: The cost of the measurement of a variable $v$ is denoted $\operatorname{cost}(v)$. By extension, the cost of the measurement of a set of variables $V$ is defined as $\operatorname{cost}(V)=$ $\sum_{v \in V} \operatorname{cost}(v)$.

Adding sensors amounts to adding terminal constraints (see Definition 7). Indeed, as mentioned in Section 3, a sensor measuring a variable $v$ is modelled by the constraint $\operatorname{val}(t, v)=v$, where $\operatorname{val}(t, v)$ is a datum coming from the sensor. Therefore, structurally speaking, a sensor measuring $v$ is modelled by a terminal constraint $k$ satisfying $\operatorname{var}(k)=\{v\}$. The constraint $k$ will be denoted by $k_{\text {sensor }}(v)$. By extension, the terminal constraints modelling sensors measuring variables $V$ are denoted by $K_{\text {sensor }}(V)$.

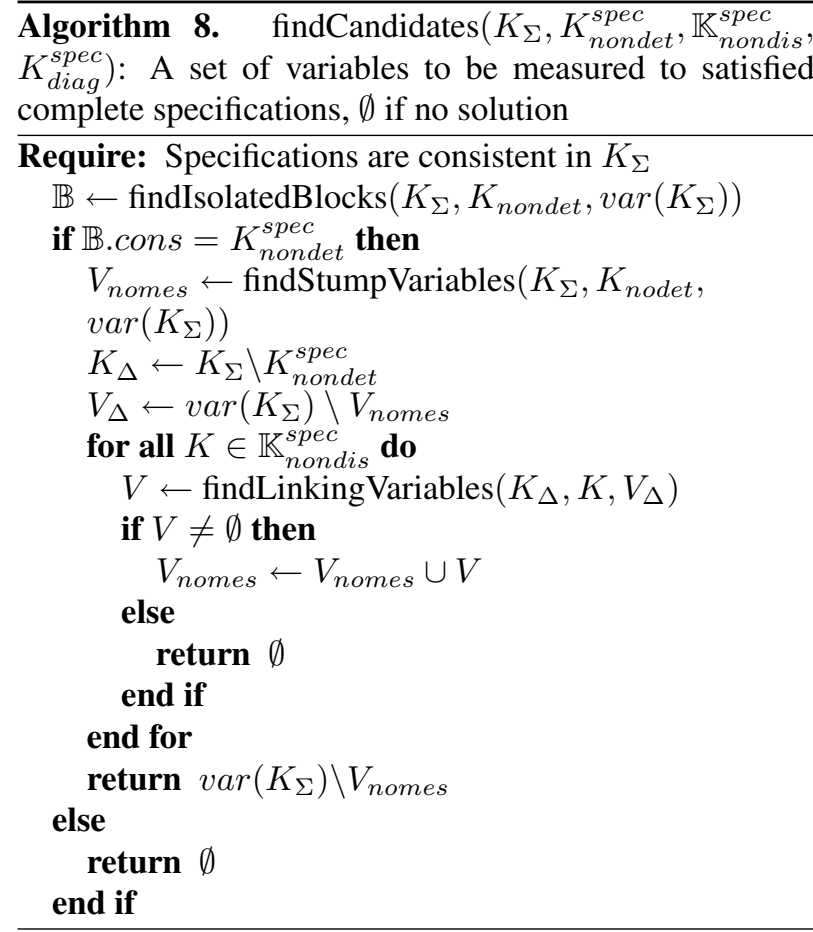

The method to solve these complete specifications can be decomposed into two steps: the determination of candidate variables for sensor placements using Theorem 1 , and the reduction of the candidate variables in order to find the minimal cost sensor placement that satisfies the complete diagnosability specifications using a branchand-bound algorithm. Figure 8 presents the dependency scheme of the method.

The findCandidates() (Algorithm 8) method is based on Theorem 1. It takes into account the specifications to determine a set of variables to be measured. If these variables are measured, the complete specifications will be satisfied. This algorithm depends on the findLinkingVariables() method, which is given by Algorithm 9. This algorithm uses the results issuing from Algorithm 5 to find a subset of variables linking a subset of constraints $K_{\Delta}$, considering only the variables $V_{\Delta}$.

In this algorithm, the cost of variables is considered. This algorithm depends on the sortVariables() method, which sorts a list of variables according to measurement costs in descending order.

A subset of the candidate variables may also lead to the satisfaction of the specifications. A branch-and-bound algorithm is used to select the most interesting candidate variables to be measured in order to find an optimal sensor placement. Before defining the optimisation algorithm, it is necessary to be able to check if the complete specifications are satisfied for a given subset of candidate variables. 

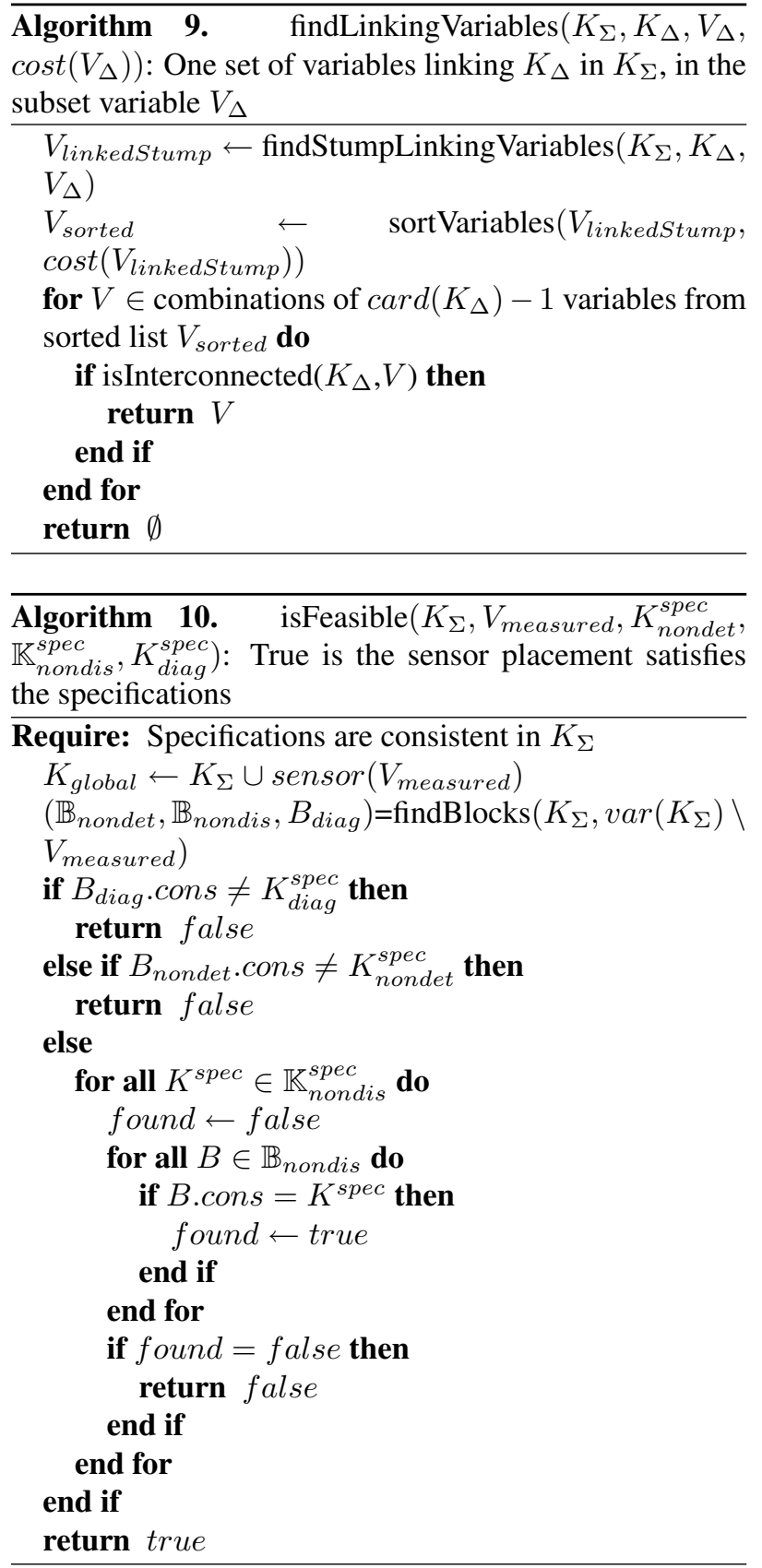

Method isCSFeasible() (Algorithm 10) achieves this.

The optimality criterion for a feasible sensor placement defined by $V_{\text {measured }}$ is given by $\operatorname{cost}\left(V_{\text {measured }}\right)$. The branch-and-bound search algorithm is implemented in the placeSensor() method (Algorithm 11) using a simple First-In First-Out buffer of nodes of variables.

\section{Application}

In this section, the special case of a dynamical system modelled by recurrent or differential equations is discussed. Then, an example is presented.

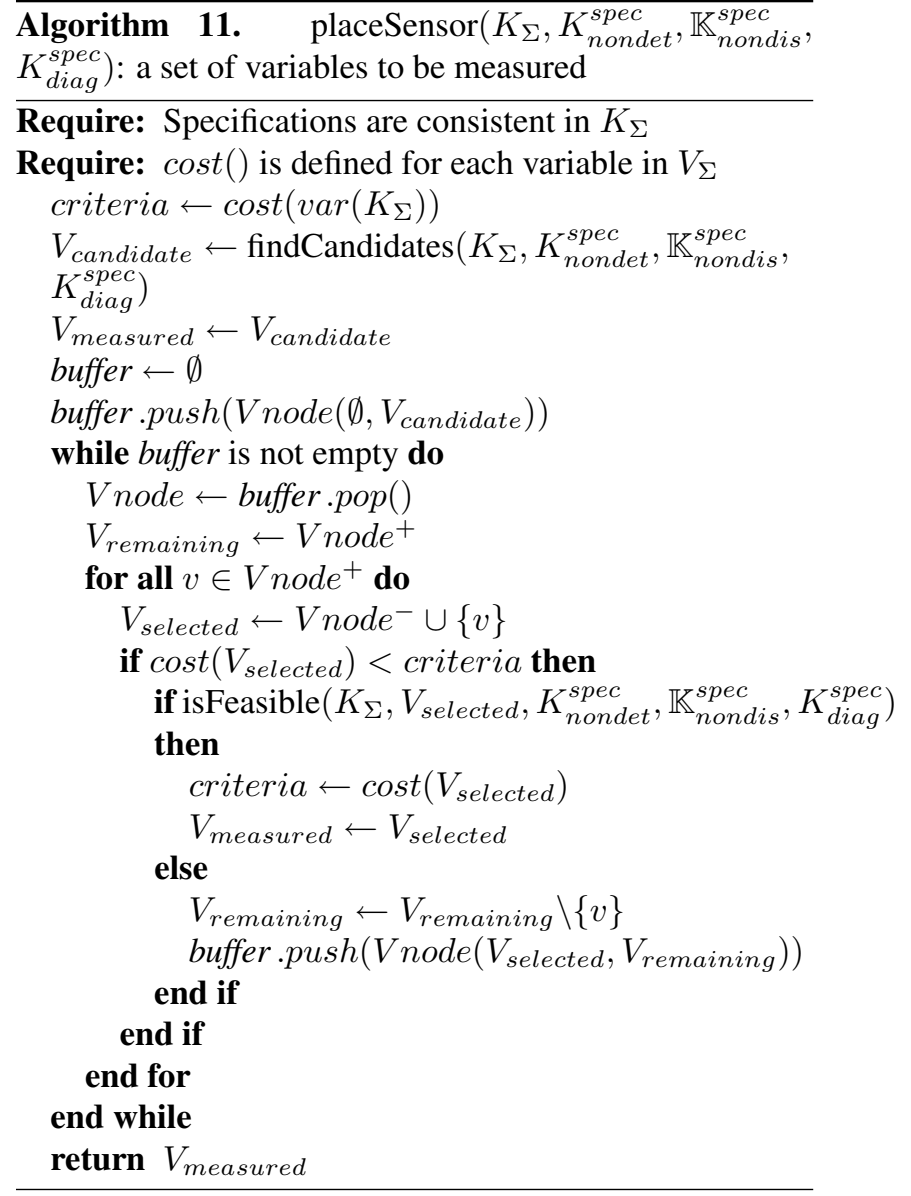

8.1. Dynamical systems. The sensor placement method relies on structural modelling. Therefore it should be suitable for most systems. Let us examine the special case of dynamical systems. Generally speaking, a model is said to be dynamic if either:

- a variable appears several times in a system but at different time, stamps, or

- a variable and some of its derivatives or summations (whatever the order is) appear in the system.

The first case mainly concerns time-delays and discrete time recurrent systems. According to Section 3, each variable stands for a tube in a phenomenological space. Therefore, a time delay, modelled by $y(t+\Delta)=x(t)$, is a constraint that establishes a link between two tubes: $\{\operatorname{dom}(y(t+\Delta)) ; \forall t\}$ and $\{\operatorname{dom}(x(t)) ; \forall t\}$. Therefore, even if the two variables model the same phenomenon, in the structural model they cannot be merged. Consider now the following discrete-time recurrent model:

$$
\begin{aligned}
x\left((k+1) T_{e}\right) & =A x\left(k T_{e}\right)+B u_{k}\left(k T_{e}\right), \\
y\left(k T_{e}\right) & =C x\left(k T_{e}\right),
\end{aligned}
$$

$k \in \mathbb{N}$, where $T_{e}$ stands for the sampling period. 
The phenomenon modelled by $x$ appears twice. Therefore, the constraint must be implicitly completed by a time delay between variables $x\left((k+1) T_{e}\right)$ and $x\left(k T_{e}\right)$. Structurally speaking, these constraints are modelled by the following structures:

$$
\begin{aligned}
& \operatorname{var}\left(k_{1}\right)=\left\{x\left(k T_{e}\right), x\left((k+1) T_{e}\right), u\left(k T_{e}\right)\right\}, \\
& \operatorname{var}\left(k_{2}\right)=\left\{x\left(k T_{e}\right), x\left((k+1) T_{e}\right)\right\} \\
& \operatorname{var}\left(k_{3}\right)=\left\{x\left(k T_{e}\right), y\left(k T_{e}\right)\right\}
\end{aligned}
$$

Moreover, if the tube corresponding to $x\left((k+1) T_{e}\right)$ appears only once in these constraints (which is usually the case in practice), constraints $k_{1}$ and $k_{2}$ can be merged:

$$
\begin{aligned}
\operatorname{var}\left(k_{12}\right) & =\left\{x\left(k T_{e}\right), u\left(k T_{e}\right)\right\}, \\
\operatorname{var}\left(k_{3}\right) & =\left\{x\left(k T_{e}\right), y\left(k T_{e}\right)\right\} .
\end{aligned}
$$

The second case mainly concerns integration and differential equations. Consider, e.g., the following model: $\frac{\mathrm{d} x}{\mathrm{~d} t}=u$. $\frac{\mathrm{d} x}{\mathrm{~d} t}$ corresponds to a tube, which can be connected to $x$ in adding the implicit constraint $x=\int \frac{\mathrm{d} x}{\mathrm{~d} t} \mathrm{~d} t$. The initial condition could also be taken into account by considering $x=\int_{0}^{t_{f}} \frac{\mathrm{d} x}{\mathrm{~d} t} \mathrm{~d} t+x_{0}$. In this case, the structures of the constraints become $\operatorname{var}\left(k_{1}\right)=\{\underline{\mathrm{d} x} \mathrm{~d} t, u\}$ and $\operatorname{var}\left(k_{2}\right)=\left\{x, \underline{\mathrm{d} x} \mathrm{~d} t, x_{0}\right\}$. In the same way as timedelays, the constraints $k_{1}$ and $k_{2}$ can be merged to obtain the following structure: $\operatorname{var}\left(k_{12}\right)=\{u, x\}$ or, if the initial condition is considered, $\operatorname{var}\left(k_{12}\right)=\left\{u, x, x_{0}\right\}$. This result remains true for summations and derivatives of any order.

Consequently, these kinds of dynamical systems can be handled just like other systems.

8.2. Example. The method presented in this paper has been applied to a sensor placement for an electronic circuit (Fig. 9). It is modelled by the following constraints:

$$
\begin{array}{llrl}
k_{1}: & v_{1 c}=v_{2}, & k_{8}: & v_{3}-v_{4 a}=R_{2} i_{2} \\
k_{2}: & i_{1}=i_{2}+i_{3}, & k_{9}: & v_{1}-v_{4 b}=R_{3} i_{3} \\
k_{3}: & v_{1}=v_{1 a}, & k_{10}: & v_{2}=R_{4} i_{4} \\
k_{4}: & v_{1}=v_{1 b}, & k_{11}: & v_{4}=v_{4 a} \\
k_{5}: & v_{1}=v_{1 c}, & k_{12}: & v_{4}=v_{4 b} \\
k_{6}: & v_{0}-v_{1}=R_{1} i_{1}, & k_{13}: & v_{0}=\operatorname{val}\left(v_{0}\right) \\
k_{7}: & C\left(v_{1 a}-v_{3}\right)=\int_{0}^{t} i_{2} \mathrm{~d} t,
\end{array}
$$

with $K_{\Sigma}=\left\{k_{1}, \ldots, k_{13}\right\}$.

The corresponding structural matrix is given by Table 1 .

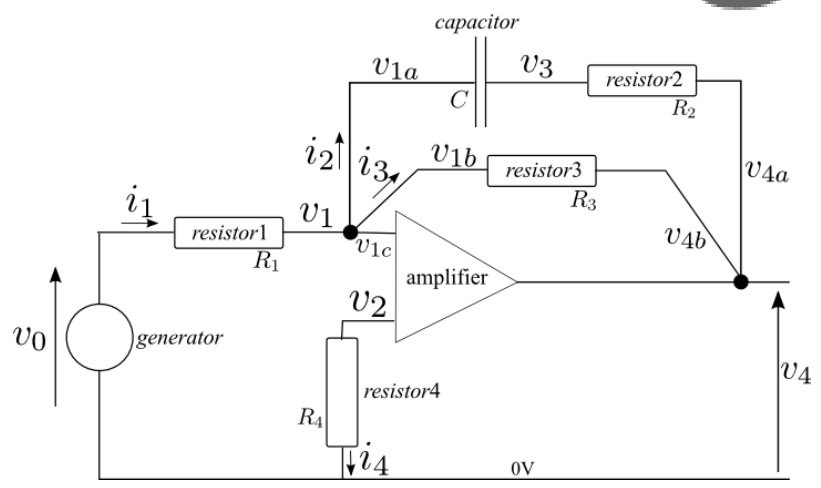

Fig. 9. Scheme of an electronic circuit.

Suppose that the costs of the measurements are

$$
\begin{array}{r}
\operatorname{cost}\left(v_{0}\right)=\operatorname{cost}\left(v_{1}\right)=\operatorname{cost}\left(v_{2}\right)=\operatorname{cost}\left(v_{3}\right)=\operatorname{cost}\left(v_{4}\right) \\
=\ldots=\operatorname{cost}\left(v_{1 a}\right)=\operatorname{cost}\left(v_{1 b}\right)=\operatorname{cost}\left(v_{1 c}\right) \\
=\operatorname{cost}\left(v_{4 a}\right)=\ldots=\operatorname{cost}\left(v_{4 b}\right)=1
\end{array}
$$

and

$$
\operatorname{cost}\left(i_{1}\right)=\operatorname{cost}\left(i_{2}\right)=\operatorname{cost}\left(i_{3}\right)=\operatorname{cost}\left(i_{4}\right)=2 .
$$

Consider the following complete specifications:

$$
\begin{aligned}
\mathbb{K}_{\text {nondis }} & =\left\{\left\{k_{2}, k_{6}\right\},\left\{k_{7}, k_{8}\right\}\right\}, \\
K_{\text {nondet }} & =\left\{k_{1}, k_{4}, k_{10}\right\}, \\
K_{\text {diag }} & =\left\{k_{3}, k_{5}, k_{9}, k_{11}, k_{12}\right\} .
\end{aligned}
$$

In order to check if the specifications $K_{\text {nondet }}$ are satisfiable, Algorithm 2 is used with $K_{\Delta}=\left\{k_{1}, k_{4}, k_{10}\right\}$, $K_{\Sigma}$ and $V_{\Delta}=\operatorname{var}\left(K_{\Sigma}\right)$. Algorithm 2 computes the following sets of isolated constraints: $\left\{\left\{k_{10}, k_{1}\right\},\left\{k_{4}\right\}\right\}$. The specifications $K_{\text {nondet }}$ are consequently satisfiable. Algorithm 2 also provides the isolated variables $V_{\text {isolated }}=\left\{i_{4}, v_{1 b}, v_{2}\right\}$.

In order to check if the specifications $\mathbb{K}_{\text {nondis }}$ are satisfiable, Algorithm 9 is used with two subsets, $K_{\Delta_{1}}=$ $\left\{k_{2}, k_{6}\right\}$ and $K_{\Delta_{2}}=\left\{k_{7}, k_{8}\right\}$, considering $V_{\Delta}=$ $\operatorname{var}\left(K_{\Sigma} \backslash V_{\text {isolated }}\right)$. Algorithm 9 computes the linking variable subsets $V_{1}=\left\{i_{1}\right\}$ and $V_{2}=\left\{v_{3}\right\}$.

In order to find the candidate variables to be measured to satisfy the specifications, Algorithm 8 is used. It yields terminal constraints that correspond to the measurements of variables $\left\{v_{0}, v_{1}, v_{4}, i_{2}, i_{3}, v_{1 a}, v_{1 c}, v_{4 a}, v_{4 b}\right\}$.

In order to find the cheapest sensor placement that satisfies the specifications, Algorithm 11 is used. It yields $V_{\text {minimal }}=\left\{v_{0}, v_{4}, i_{2}, i_{3}, v_{1 a}, v_{1 c}, v_{4 a}, v_{4 b}\right\}$ with a cost of 10 .

In order to validate the result, the method proposed in (Ploix et al., 2005) has been used to design all the ARRs. It has led to the fault signature given by Table 2 . 
Table 1. Structural matrix of the electronic circuit.

\begin{tabular}{|c|c|c|c|c|c|c|c|c|c|c|c|c|c|c|}
\hline & $v_{0}$ & $v_{1}$ & $v_{2}$ & $v_{3}$ & $v_{4}$ & $i_{1}$ & $i_{2}$ & $i_{3}$ & $i_{4}$ & $v_{1 a}$ & $v_{1 b}$ & $v_{1 c}$ & $v_{4 a}$ & $v_{4 b}$ \\
\hline \hline$k_{1}$ & 0 & 0 & 1 & 0 & 0 & 0 & 0 & 0 & 0 & 0 & 0 & 1 & 0 & 0 \\
\hline$k_{2}$ & 0 & 0 & 0 & 0 & 0 & 1 & 1 & 1 & 0 & 0 & 0 & 0 & 0 & 0 \\
\hline$k_{3}$ & 0 & 1 & 0 & 0 & 0 & 0 & 0 & 0 & 0 & 1 & 0 & 0 & 0 & 0 \\
\hline$k_{4}$ & 0 & 1 & 0 & 0 & 0 & 0 & 0 & 0 & 0 & 0 & 1 & 0 & 0 & 0 \\
\hline$k_{5}$ & 0 & 1 & 0 & 0 & 0 & 0 & 0 & 0 & 0 & 0 & 0 & 1 & 0 & 0 \\
\hline$k_{6}$ & 1 & 1 & 0 & 0 & 0 & 1 & 0 & 0 & 0 & 0 & 0 & 0 & 0 & 0 \\
\hline$k_{7}$ & 0 & 0 & 0 & 1 & 0 & 0 & 1 & 0 & 0 & 1 & 0 & 0 & 0 & 0 \\
\hline$k_{8}$ & 0 & 0 & 0 & 1 & 0 & 0 & 1 & 0 & 0 & 0 & 0 & 0 & 1 & 0 \\
\hline$k_{9}$ & 0 & 1 & 0 & 0 & 0 & 0 & 0 & 1 & 0 & 0 & 0 & 0 & 0 & 1 \\
\hline$k_{10}$ & 0 & 0 & 1 & 0 & 0 & 0 & 0 & 0 & 1 & 0 & 0 & 0 & 0 & 0 \\
\hline$k_{11}$ & 0 & 0 & 0 & 0 & 1 & 0 & 0 & 0 & 0 & 0 & 0 & 0 & 1 & 0 \\
\hline$k_{12}$ & 0 & 0 & 0 & 0 & 1 & 0 & 0 & 0 & 0 & 0 & 0 & 0 & 0 & 1 \\
\hline$k_{13}$ & 1 & 0 & 0 & 0 & 0 & 0 & 0 & 0 & 0 & 0 & 0 & 0 & 0 & 0 \\
\hline
\end{tabular}

Table 2. Analytical relations for the complete specifications.

\begin{tabular}{|c|c|c|c|c|c|c|c|c|c|c|c|c|c|}
\hline & $k_{1}$ & $k_{2}$ & $k_{3}$ & $k_{4}$ & $k_{5}$ & $k_{6}$ & $k_{7}$ & $k_{8}$ & $k_{9}$ & $k_{10}$ & $k_{11}$ & $k_{12}$ & $k_{13}$ \\
\hline \hline$A R R_{1}$ & 0 & 1 & 0 & 0 & 0 & 1 & 0 & 0 & 1 & 0 & 0 & 0 & 1 \\
\hline$A R R_{2}$ & 0 & 1 & 0 & 0 & 0 & 1 & 0 & 0 & 1 & 0 & 0 & 0 & 0 \\
\hline$A R R_{3}$ & 0 & 1 & 1 & 0 & 0 & 1 & 0 & 0 & 0 & 0 & 0 & 0 & 1 \\
\hline$A R R_{4}$ & 0 & 1 & 1 & 0 & 0 & 1 & 0 & 0 & 0 & 0 & 0 & 0 & 0 \\
\hline$A R R_{5}$ & 0 & 0 & 1 & 0 & 0 & 0 & 0 & 0 & 1 & 0 & 0 & 0 & 0 \\
\hline$A R R_{6}$ & 0 & 1 & 0 & 0 & 1 & 1 & 0 & 0 & 0 & 0 & 0 & 0 & 1 \\
\hline$A R R_{7}$ & 0 & 0 & 0 & 0 & 0 & 0 & 1 & 1 & 0 & 0 & 0 & 0 & 0 \\
\hline$A R R_{8}$ & 0 & 1 & 0 & 0 & 1 & 1 & 0 & 0 & 0 & 0 & 0 & 0 & 0 \\
\hline$A R R_{9}$ & 0 & 0 & 0 & 0 & 0 & 0 & 0 & 0 & 0 & 0 & 0 & 0 & 1 \\
\hline$A R R_{10}$ & 0 & 0 & 0 & 0 & 0 & 0 & 0 & 0 & 0 & 0 & 1 & 0 & 0 \\
\hline$A R R_{11}$ & 0 & 0 & 0 & 0 & 1 & 0 & 0 & 0 & 1 & 0 & 0 & 0 & 0 \\
\hline$A R R_{12}$ & 0 & 0 & 1 & 0 & 1 & 0 & 0 & 0 & 0 & 0 & 0 & 0 & 0 \\
\hline$A R R_{13}$ & 0 & 0 & 0 & 0 & 0 & 0 & 0 & 0 & 0 & 0 & 0 & 1 & 0 \\
\hline
\end{tabular}

According to these results, the constraint sets that cannot be discriminated are $\left\{k_{2}, k_{6}\right\}$ and $\left\{k_{7}, k_{8}\right\}$. The constraint set that cannot be detected is $\left\{k_{1}, k_{4}, k_{10}\right\}$ and the diagnosable constraints are $\left\{k_{3}, k_{5}, k_{9}, k_{11}, k_{12}\right\}$. Applying the function $\Phi: K_{\Sigma} \longrightarrow C_{\Sigma}$, it is obvious that the components that cannot be discriminated are $\left\{c_{2}, c_{6}\right\}$ and $\left\{c_{7}, c_{8}\right\}$, the components that cannot be detected are $\left\{c_{1}, c_{4}, c_{10}\right\}$, and the diagnosable components are $\left\{c_{3}, c_{5}, c_{9}, c_{11}, c_{12}\right\}$.

Suppose now that the specifications are given by

$$
\begin{aligned}
\mathbb{K}_{\text {nondis }} & =\left\{\left\{k_{2}, k_{3}\right\},\left\{k_{7}, k_{8}\right\}\right\}, \\
K_{\text {nondet }} & =\left\{k_{1}, k_{10}\right\}, \\
K_{\text {diag }} & =\left\{k_{6}, k_{4}, k_{5}, k_{9}, k_{11}, k_{12}\right\} .
\end{aligned}
$$

In order to check if the specifications $K_{\text {nondet }}$ are satisfiable, Algorithm 2 is used with $K_{\Delta}=\left\{k_{1}, k_{10}\right\}, K_{\Sigma}$ and
$V_{\Delta}=\operatorname{var}\left(K_{\Sigma}\right)$. Algorithm 2 computes the following sets of isolated constraints: $\left\{\left\{k_{10}, k_{1}\right\}\right\}$. The specifications $K_{\text {nondet }}$ are consequently satisfiable. Algorithm 2 also provides the isolating variables $V_{\text {isolated }}=\left\{i_{4}, v_{2}\right\}$.

In order to check if the specifications $\mathbb{K}_{\text {nondis }}$ are satisfiable, Algorithm 9 is used with the two subsets $K_{\Delta_{1}}=\left\{k_{2}, k_{3}\right\}$ and $K_{\Delta_{2}}=\left\{k_{7}, k_{8}\right\}$, considering $V_{\Delta}=\operatorname{var}\left(K_{\Sigma} \backslash V_{\text {isolated }}\right)$. Because Algorithm 9 computes the linking variable subset $V_{1}=\{\emptyset\}$ for the constraint subset $K_{\Delta_{1}}=\left\{k_{2}, k_{3}\right\}$, there is no solution that satisfies these specifications.

The results presented in this paper demonstrate that it is possible to design optimal sensor placements satisfying diagnosability criteria without designing the ARR $a$ priori. 


\section{Conclusion}

A new approach to sensor placement has been proposed that makes it possible to satisfy diagnosability specifications. It is thus possible to specify the performances that a diagnostic system has to meet and then to compute where the sensors should be placed.

The presented lemmas, theorems and algorithms are general and can be reused to develop other methods for sensor placement that deal with various kinds of specifications, e.g., a set of components that have to be at least detectable and another one of those that have to be diagnosable. The provided tools apply to any system including dynamical systems described by recurrent or differential equations because they are based on a structural approach: only the variables appearing in constraints are considered. However, the generality of the structural approach is paid by possible over-estimation depending on the nature of constraints: it is well known that it relies on the conditioning of constraints. But solutions taking into account the nature of constraints can only be specific.

An algorithm for sensor placement managing complete specifications has been presented. It deals with elements that have to be diagnosable, discriminable and nondetectable. Thanks to the proposed algorithm, cost optimal sensor placement satisfying complete diagnosability specifications is possible without designing the ARR $a$ priori. This is a very important feature since it is no longer necessary to design all the possible ARRs assuming all the variables are measured.

This approach manages only specifications dealing with models of the normal behaviour. It does not take into account specific fault models such as a leak in a pipe. Therefore, if such models are considered, the sensor placement algorithm will lead to an over-estimation of the required sensors. Taking into account specific fault models may lead to a reduction of the required sensors. Nevertheless, fault models cannot be easily taken into account in sensor placement methods. It is still an open problem.

\section{References}

Apt K. (2003). Principles of Constraint Programming, Cambridge University Press, Amsterdam, The Netherlands.

Blanke M., Kinnaert M., Lunze J. and Staroswiecki, M. (2006). Diagnosis and Fault-tolerant Control, Springer-Verlag, Berlin/Heidelberg.

Bollobás B. (1998). Modern Graph Theory, Springer, New York, NY.

Chittaro L. and Ranon R. (2004). Hierarchical model-based diagnosis based on structural abstraction, Artificial Intelligence 155(1-2): 147-182.

Commault C., Dion J.-M. and Yacoub Agha S. (2006). Structural analysis for the sensor location problem in fault detection and isolation, Proceedings of the IFAC Symposium SAFEPROCESS'2006, Beijing, China, CD-ROM.
Console L., Picardi C. and Ribando M. (2000). Diagnosis and diagnosability analysis using process algebra, Proceedings of the 11th International Workshop on Principles of Diagnosis DX'2000, Morelia, Mexico.

Cordier M.-O., Dague P., Lévy F., Dumas M., Montmain J., Staroswiecki, M. and Travé-Massuyès, L. (2000). A comparative analysis of AI and control theory approaches to model-based diagnosis, Proceedings of the IFAC Symposium SAFEPROCESS 2000, Budapest, Hungary, pp. 329334.

Dalton T., Klotzek P. and Frank P. (1999). Application of sensitivity theory to fuzzy logic based FDI, International Journal of Applied Mathematics and Computer Science 9(3): 619-636.

de Kleer J. and Williams B. C. (1992). Diagnosis with behavioral modes, in (W. Hamscher, L. Console and J. de Kleer, Eds.), Readings in Model-Based Diagnosis, Morgan Kaufmann Publishers Inc., San Francisco, CA, pp. 124-130.

Dulmage A. L. and Mendelsohn N. S. (1959). A structure theory of bi-partite graphs of finite exterior extension, Transactions of the Royal Society of Canada 53(III): 1-13.

Frisk E. and Krysander M. (2007). Sensor placement for maximum fault isolability, Proceedings of the 18th International Workshop on Principles of Diagnosis (DX-07), Nashville, TN, USA.

Hamscher W., Console L. and De Kleer J. (1992). Readings in Model-Based Diagnosis, Morgan Kaufmann Publishers Inc., San Francisco, CA.

Korbicz J., Patan K. and Obuchowicz A. (1999). Dynamic neural networks for process modelling in fault detection and isolation, International Journal of Applied Mathematics and Computer Science 9(3): 519-546.

Koscielny J., Syfert M. and Bartys M. (1999). Fuzzy logic faut diagnosis of industrial process actuators, International Journal of Applied Mathematics and Computer Science 9(3): 653-666.

Krysander M., Aslund J. and Nyberg M. (2008). An efficient algorithm for finding minimal overconstrained subsystems for model-based diagnosis, IEEE Transactions on Systems, Man and Cybernetics, Part A 38(1): 197-206.

Lopez-Toribio C., Patton R. and Uppal F. (1999). Artificial intelligence approaches to fault diagnosis for dynamic systems, International Journal of Applied Mathematics and Computer Science 9(3): 471-518.

Madron F. and Veverka V. (1992). Optimal selection of measuring points in complex plants by linear models, AIChE Journal 38(2): 227-236.

Maquin D., Luong M. and Ragot J. (1997). Fault detection and isolation and sensor network design, European Journal of Automation 31(2): 393-406.

Nyberg M. and Krysander M. (2003). Combining AI, FDI, and statistical hypothesis-testing in a framework for diagnosis, Proceedings of the IFAC Symposium SAFEPROCESS'03, Washington, DC, USA, pp. 813-818. 
Ploix S., Désinde M. and Touaf S. (2005). Automatic design of detection tests in complex dynamic systems, Proceedings of the 16th IFAC World Congress, Prague, Czech Republic.

Ploix S., Touaf S. and Flaus J. M. (2003). A logical framework for isolation in fault diagnosis, Proceedings of the IFAC Symposium SAFEPROCESS'2003, Washington, DC, USA.

Pothen A. and Chin-Ju F. (1990). Computing the block triangular form of a sparse matrix, ACM Transactions on Mathematical Software 16(4): 303-324.

Pulido B. and Alonso C. (2002). Possible conflicts, arrs, and conflicts, Proceedings of the 13th International Workshop on Principles of Diagnosis (DX02), Semmering, Austria, pp. 122-128.

Shumsky A. (2007). Redundancy relations for fault diagnosis in nonlinear uncertain systems, International Journal of Applied Mathematics and Computer Science 17(4): 477-489.

Struss P. (1992). What's in SD? Towards a theory of modeling for diagnosis, in (W. Hamscher, L. Console and J. de Kleer, Eds.), Readings in model-based diagnosis, Morgan Kaufmann Publishers Inc., San Francisco, CA, pp. 419-449. .
Struss P., Rehfus B., Brignolo R., Cascio F., Console L., Dague P., Dubois P., Dressler O. and Millet D. (2002). Modelbased tools for the integration of design and diagnosis into a common process - A project report, Proceedings of the International Workshop on Principles of Diagnosis DX'02, Semmering, Austria, pp. 25-32.

Travé-Massuyès L., Escobet T. and Milne R. (2001). Modelbased diagnosability and sensor placement application to a frame 6 gas turbine subsystem, Proceedings of the 12th International Workshop on Principles of Diagnosis, Sansicario, Via Lattea, Italy, pp. 205-212.

Witczak M. (2006). Advances in model-based fault diagnosis with evolutionary algorithms and neural networks, International Journal of Applied Mathematics and Computer Science 16(1): 85-99.

Received: 2 December 2007

Revised: 14 May 2008 